Supporting Information

\title{
Thiolate-Bridged Nickel-Iron and Nickel-Ruthenium Complexes Relevant to the CO-inhibited State of [NiFe]-hydrogenase
}

Puhua Sun, Dawei Yang, Ying Li, Yahui Zhang, Linan Su, Baomin Wang, and Jingping $\mathrm{Qu}^{*}$

State Key Laboratory of Fine Chemicals, School of Pharmaceutical Science and Technology, Faculty of Chemical, Environmental and Biological Science and Technology, Dalian University of Technology, 2 Linggong Road, Dalian 116024, P. R. China

*E-mail: qujp@dlut.edu.cn 


\section{Contents:}

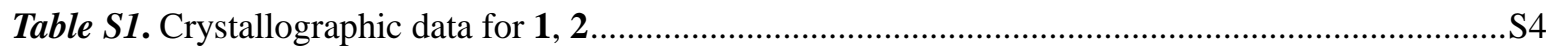

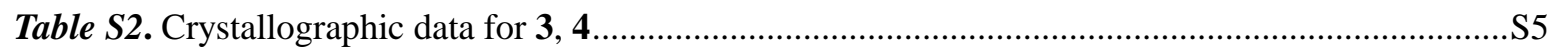

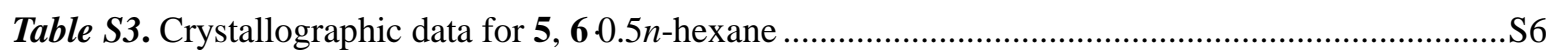

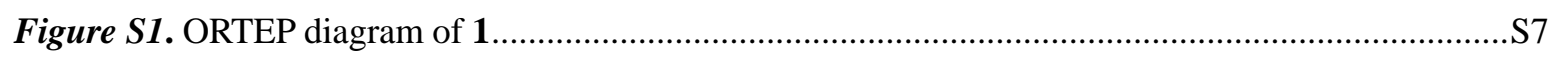

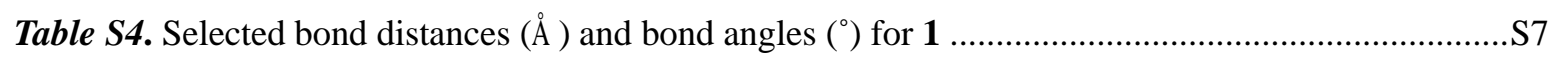

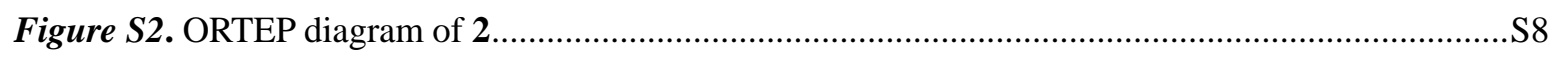

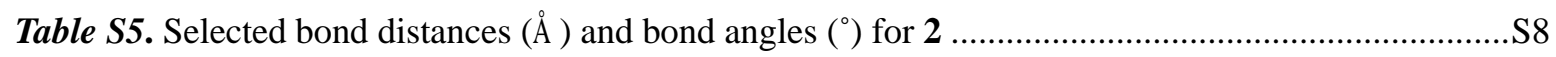

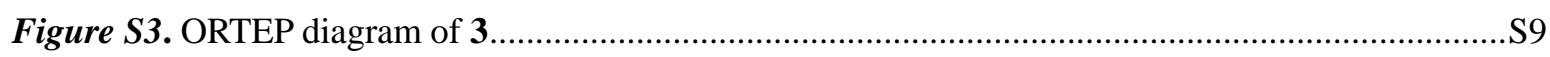

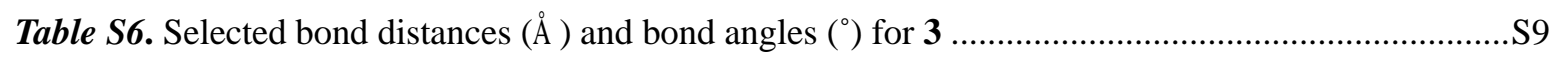

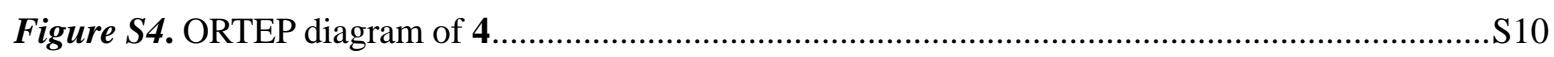

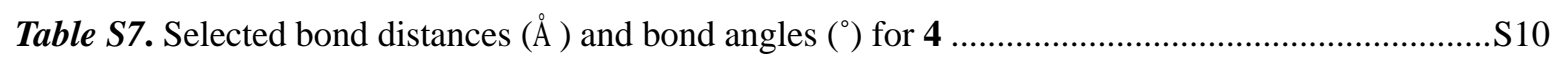

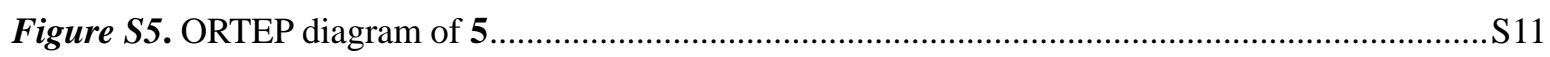

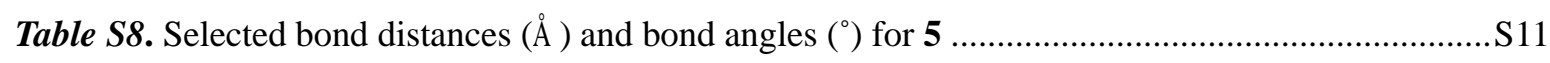

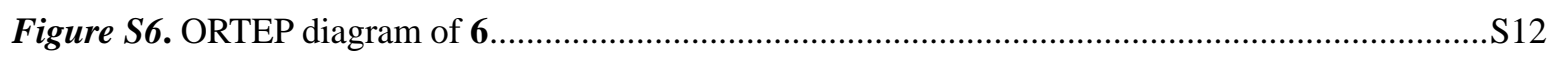

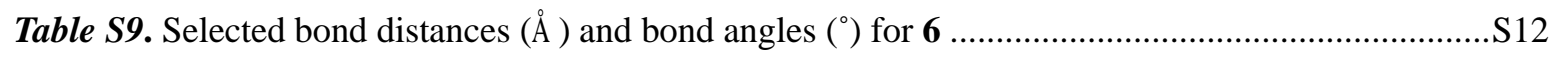

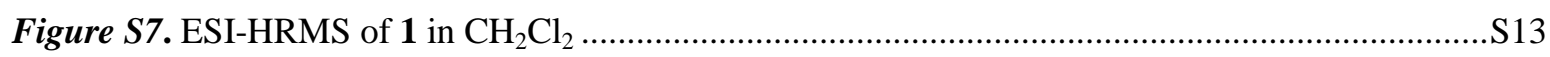

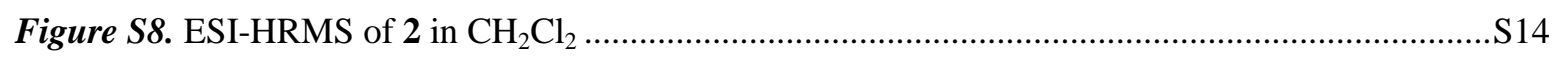

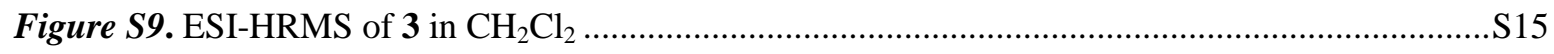

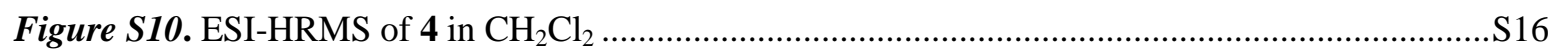

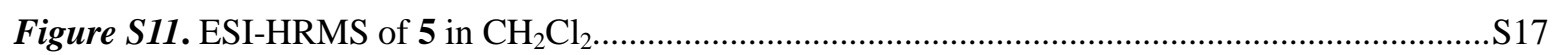

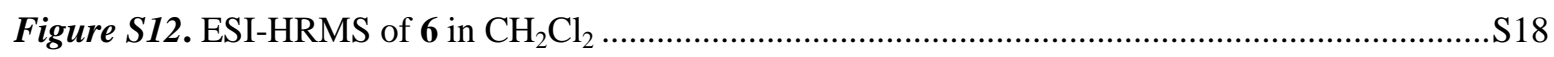

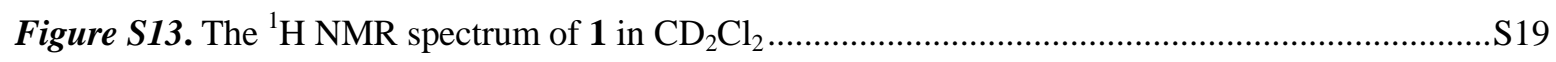

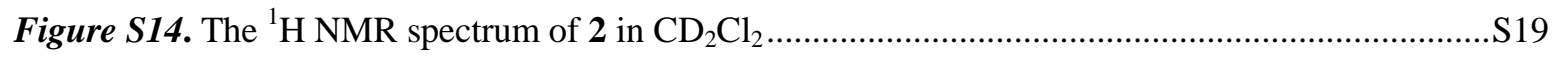

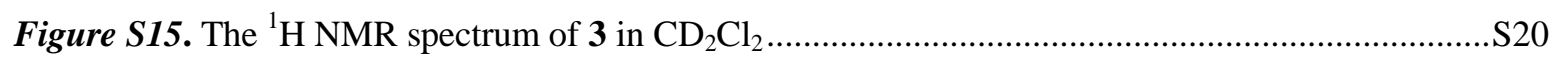

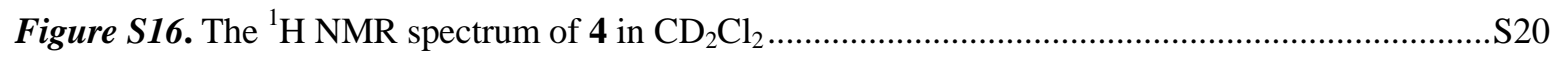

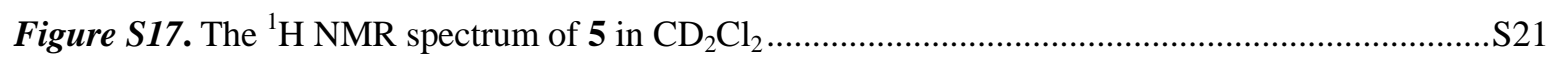

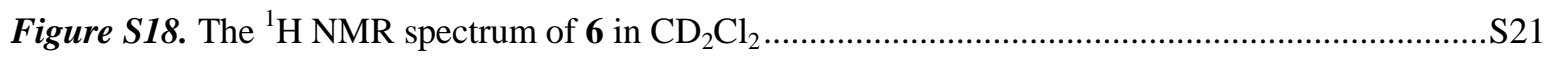

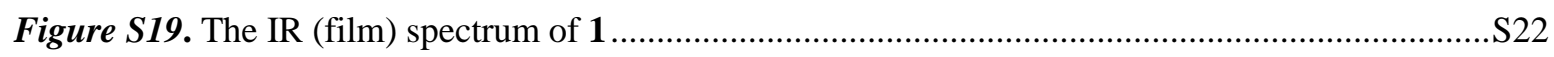

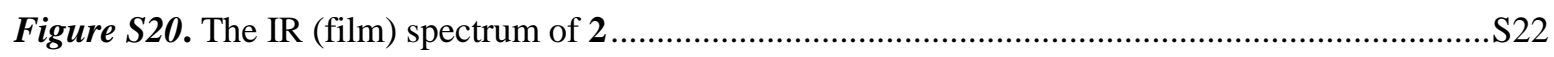

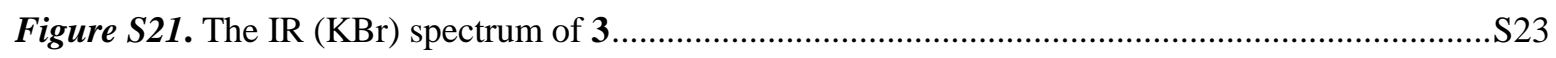

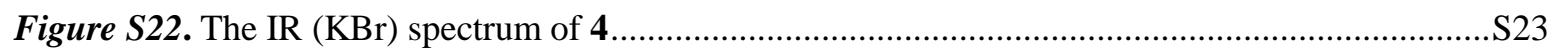

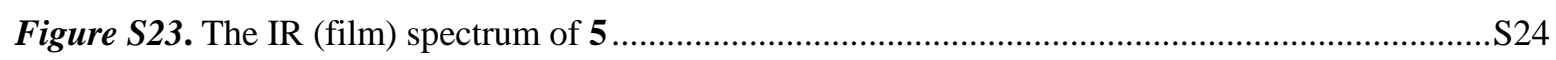

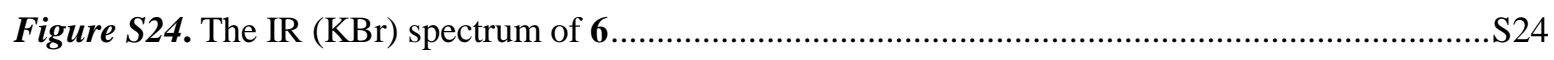

Figure S25. The cyclic voltammogram of $1(1 \mathrm{mM})$ in $0.1 \mathrm{M}^{n} \mathrm{Bu}_{4} \mathrm{NPF}_{6} / \mathrm{CH}_{2} \mathrm{Cl}_{2}$ at $25^{\circ} \mathrm{C}$ with a scan rate of $100 \mathrm{mV} \mathrm{s}^{-1}$

Figure S26. The cyclic voltammograms of $1(1 \mathrm{mM})$ in $0.1 \mathrm{M}^{n} \mathrm{Bu}_{4} \mathrm{NPF}_{6} / \mathrm{CH}_{2} \mathrm{Cl}_{2}$ at $25^{\circ} \mathrm{C}$ at various scan

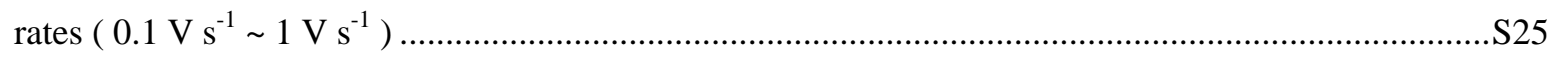

Figure S27. The cyclic voltammogram of 1 in the presence of 1 equivalent of $\left[\mathrm{HEt}_{3} \mathrm{~N}\right]\left[\mathrm{BF}_{4}\right]$ in $0.1 \mathrm{M}$

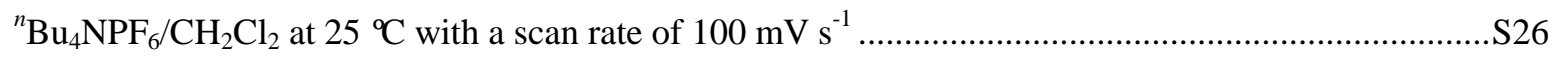

Figure S28. Cyclic voltammograms of $1\left(1 \mathrm{mM}\right.$ in $0.1 \mathrm{M}^{n} \mathrm{Bu}_{4} \mathrm{NPF}_{6}$ in $\mathrm{CH}_{2} \mathrm{Cl}_{2}$ under Ar) with

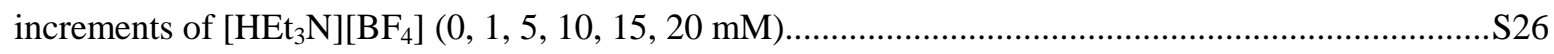

Figure S29. The cyclic voltammogram of $2(1 \mathrm{mM})$ in $0.1 \mathrm{M}^{n} \mathrm{Bu}_{4} \mathrm{NPF}_{6} / \mathrm{CH}_{2} \mathrm{Cl}_{2}$ at $25^{\circ} \mathrm{C}$ with a scan rate 
of $100 \mathrm{mV} \mathrm{s}^{-1}$

Figure S30. The cyclic voltammograms of $2(1 \mathrm{mM})$ in $0.1 \mathrm{M}^{n} \mathrm{Bu}_{4} \mathrm{NPF}_{6} / \mathrm{CH}_{2} \mathrm{Cl}_{2}$ at $25^{\circ} \mathrm{C}$ at various scan rates $\left(0.1 \mathrm{~V} \mathrm{~s}^{-1} \sim 1 \mathrm{~V} \mathrm{~s}^{-1}\right)$

Figure S31. The cyclic voltammogram of 2 in the presence of 1 equivalent of $\left[\mathrm{HEt}_{3} \mathrm{~N}_{[}\left[\mathrm{BF}_{4}\right]\right.$ in $0.1 \mathrm{M}$ ${ }^{n} \mathrm{Bu}_{4} \mathrm{NPF}_{6} / \mathrm{CH}_{2} \mathrm{Cl}_{2}$ at $25^{\circ} \mathrm{C}$ with a scan rate of $100 \mathrm{mV} \mathrm{s}{ }^{-1}$.

Figure S32. The cyclic voltammogram of $3(1 \mathrm{mM})$ in $0.1 \mathrm{M}^{n} \mathrm{Bu}_{4} \mathrm{NPF}_{6} / \mathrm{CH}_{2} \mathrm{Cl}_{2}$ at $25^{\circ} \mathrm{C}$ with a scan rate of $100 \mathrm{mV} \mathrm{s}^{-1}$

Figure S33. The cyclic voltammograms of $3(1 \mathrm{mM})$ in $0.1 \mathrm{M}^{n} \mathrm{Bu}_{4} \mathrm{NPF}_{6} / \mathrm{CH}_{2} \mathrm{Cl}_{2}$ at $25^{\circ} \mathrm{C}$ at various scan rates $\left(0.1 \mathrm{~V} \mathrm{~s}^{-1} \sim 1 \mathrm{~V} \mathrm{~s}^{-1}\right)$.

Figure S34. The cyclic voltammogram of $4(1 \mathrm{mM})$ in $0.1 \mathrm{M}^{n} \mathrm{Bu}_{4} \mathrm{NPF}_{6} / \mathrm{CH}_{2} \mathrm{Cl}_{2}$ at $25^{\circ} \mathrm{C}$ with a scan rate of $100 \mathrm{mV} \mathrm{s}^{-1}$

Figure S35. The cyclic voltammograms of $4(1 \mathrm{mM})$ in $0.1 \mathrm{M}^{n} \mathrm{Bu}_{4} \mathrm{NPF}_{6} / \mathrm{CH}_{2} \mathrm{Cl}_{2}$ at $25^{\circ} \mathrm{C}$ at various scan rates $\left(0.1 \mathrm{~V} \mathrm{~s}^{-1} \sim 1 \mathrm{~V} \mathrm{~s}^{-1}\right)$

Figure S36. The cyclic voltammogram of $5(1 \mathrm{mM})$ in $0.1 \mathrm{M}^{n} \mathrm{Bu}_{4} \mathrm{NPF}_{6} / \mathrm{CH}_{2} \mathrm{Cl}_{2}$ at $25^{\circ} \mathrm{C}$ with a scan rate of $100 \mathrm{mV} \mathrm{s}^{-1}$

Figure S37. The cyclic voltammogram of $6(1 \mathrm{mM})$ in $0.1 \mathrm{M}^{n} \mathrm{Bu}_{4} \mathrm{NPF}_{6} / \mathrm{CH}_{2} \mathrm{Cl}_{2}$ at $25^{\circ} \mathrm{C}$ with a scan rate of $100 \mathrm{mV} \mathrm{s}^{-1}$ S31

Figure S38. Corresponding plot of $\mathrm{i}_{\mathrm{c}} / \mathrm{i}_{\mathrm{p}}$ vs acid concentration S31 
Table S1. Crystallographic data for 1, 2

\begin{tabular}{lcc}
\hline & $\mathbf{1}$ & $\mathbf{2}$ \\
\hline Formula & $\mathrm{C}_{40} \mathrm{H}_{47} \mathrm{~F}_{6} \mathrm{FeNiP}_{3} \mathrm{~S}_{3}$ & $\mathrm{C}_{40} \mathrm{H}_{47} \mathrm{~F}_{6} \mathrm{FeNiOP}_{3} \mathrm{~S}_{2}$ \\
Formula weight & 945.43 & 929.37 \\
Crystal dimensions $\left(\mathrm{mm}^{3}\right)$ & $0.29 \times 0.27 \times 0.25$ & $0.28 \times 0.26 \times 0.23$ \\
Crystal system & Monoclinic & Monoclinic \\
Space group & $\mathrm{C} 2 / \mathrm{c}$ & $\mathrm{C} 2 / \mathrm{c}$ \\
$\mathrm{a}(\AA)$ & $19.4970(15)$ & $19.427(4)$ \\
$\mathrm{b}(\AA)$ & $13.3056(11)$ & $13.236(3)$ \\
$\mathrm{c}(\AA)$ & $35.574(3)$ & $35.630(8)$ \\
$\alpha\left(^{\circ}\right)$ & 90.00 & 90.00 \\
$\beta\left({ }^{\circ}\right)$ & $102.8730(10)$ & $102.823(3)$ \\
$\gamma\left({ }^{\circ}\right)$ & 90.00 & 90.00 \\
Volume $\left(\AA^{3}\right)$ & $8996.7(13)$ & $8934(3)$ \\
$Z$ & 8 & 8 \\
$T(\mathrm{~K})$ & $298(2)$ & $298(2)$ \\
$D$ calcd $(\mathrm{g}$ cm & -3 \\
$\mu\left(\mathrm{mm}{ }^{-1}\right)$ & 1.396 & 1.382 \\
$F(000)$ & 1.039 & 1.002 \\
No. of rflns. collected & 3904 & 3840 \\
No. of indep. rflns. $/ R_{\text {int }}$ & 23323 & 23482 \\
No. of obsd. rflns. $\left[I_{0}>2 \sigma\left(I_{0}\right)\right]$ & $7873 / 0.0334$ & $7838 / 0.0341$ \\
Data / restraints / parameters & 5963 & 5894 \\
$R_{l} / w R_{2}\left[I_{0}>2 \sigma\left(I_{0}\right)\right]^{\mathrm{a}}$ & $7873 / 0 / 519$ & $7838 / 91 / 557$ \\
$R_{I} / w R_{2}(\text { all data })^{\mathrm{a}}$ & $0.0604 / 0.1743$ & $0.0585 / 0.1825$ \\
$\mathrm{GOF}\left(\text { on } F^{2}\right)^{\mathrm{a}}$ & $0.0796 / 0.1863$ & $0.0786 / 0.1954$ \\
Largest diff. peak and hole $\left(\mathrm{e} \AA^{-3}\right)$ & 1.008 & 1.080 \\
& $0.816 /-0.318$ & $0.744 /-0.405$ \\
\hline
\end{tabular}


Table S2. Crystallographic data for 3,4

\begin{tabular}{lcc}
\hline & $\mathbf{3}$ & $\mathbf{4}$ \\
\hline Formula & $\mathrm{C}_{40} \mathrm{H}_{47} \mathrm{~F}_{6} \mathrm{RuNiP}_{3} \mathrm{~S}_{3}$ & $\mathrm{C}_{40} \mathrm{H}_{47} \mathrm{~F}_{6} \mathrm{RuNiOP}_{3} \mathrm{~S}_{2}$ \\
Formula weight & 990.65 & 902.10 \\
Crystal dimensions $\left(\mathrm{mm}^{3}\right)$ & $0.29 \times 0.27 \times 0.25$ & $0.28 \times 0.26 \times 0.24$ \\
Crystal system & Monoclinic & Monoclinic \\
Space group & $\mathrm{C} 2 / \mathrm{c}$ & $\mathrm{C} 2 / \mathrm{c}$ \\
$\mathrm{a}(\AA)$ & $19.1698(8)$ & $19.3966(6)$ \\
$\mathrm{b}(\AA)$ & $13.5266(8)$ & $13.4689(6)$ \\
$\mathrm{c}(\AA)$ & $35.806(2)$ & $33.8884(12)$ \\
$\alpha\left(^{\circ}\right)$ & 90.00 & 90.00 \\
$\beta\left(^{\circ}\right)$ & $103.301(3)$ & $91.1632(18)$ \\
$\gamma\left({ }^{\circ}\right)$ & 90.00 & 90.00 \\
Volume $\left(\AA^{3}\right)$ & $9035.4(8)$ & $8851.6(6)$ \\
$Z$ & 8 & 8 \\
$T(\mathrm{~K})$ & $298(2)$ & $298(2)$ \\
$D$ calcd $(\mathrm{g}$ cm & -3 \\
$\left.\mu(\mathrm{mm})^{-1}\right)$ & 1.456 & 1.463 \\
$F(000)$ & 1.048 & 1.024 \\
No. of rflns. collected & 4048 & 3984 \\
No. of indep. rflns. $/ R_{\text {int }}$ & 69481 & 72801 \\
No. of obsd. rflns. $\left[I_{0}>2 \sigma\left(I_{0}\right)\right]$ & $7931 / 0.0793$ & $7742 / 0.0301$ \\
Data / restraints / parameters & 6472 & 7115 \\
$R_{l} / w R_{2}\left[I_{0}>2 \sigma\left(I_{0}\right)\right]^{\mathrm{a}}$ & $7931 / 811 / 519$ & $7742 / 108 / 519$ \\
$R_{l} / w R_{2}(\text { all data })^{\mathrm{a}}$ & $0.0689 / 0.1767$ & $0.0550 / 0.1563$ \\
$\mathrm{GOF}\left(\text { on } F^{2}\right)^{\mathrm{a}}$ & $0.0861 / 0.1862$ & $0.0600 / 0.1595$ \\
Largest diff. peak and hole $\left(\mathrm{e} \AA^{-3}\right)$ & 1.084 & 1.036 \\
& $0.862 /-0.701$ &
\end{tabular}


Table S3. Crystallographic data for 5, 6.0.5n-hexane

\begin{tabular}{lcc}
\hline & $\mathbf{5}$ & $\mathbf{6} \cdot 0.5 n$-hexane \\
\hline Formula & $\mathrm{C}_{41} \mathrm{H}_{47} \mathrm{~F}_{6} \mathrm{FeNiO}_{2} \mathrm{P}_{3} \mathrm{~S}_{2}$ & $\mathrm{C}_{44} \mathrm{H}_{54} \mathrm{~F}_{6} \mathrm{RuNiO}_{2} \mathrm{P}_{3} \mathrm{~S}_{2}$ \\
Formula weight & 957.38 & 1045.68 \\
Crystal dimensions $\left(\mathrm{mm}^{3}\right)$ & $0.30 \times 0.27 \times 0.25$ & $0.26 \times 0.25 \times 0.22$ \\
Crystal system & Monoclinic & Monoclinic \\
Space group & $\mathrm{Cc}$ & $\mathrm{Cc}$ \\
$\mathrm{a}(\AA)$ & $16.6651(16)$ & $16.7547(7)$ \\
$\mathrm{b}(\AA)$ & $16.7535(16)$ & $16.8040(7)$ \\
$\mathrm{c}(\AA)$ & $33.545(3)$ & $33.4582(14)$ \\
$\alpha\left(^{\circ}\right)$ & 90.00 & 90.00 \\
$\beta\left({ }^{\circ}\right)$ & $94.544(3)$ & $93.988(2)$ \\
$\gamma\left({ }^{\circ}\right)$ & 90.00 & 90.00 \\
Volume $\left(\AA^{3}\right)$ & $9336.3(15)$ & $9397.2(7)$ \\
$Z$ & 8 & 8 \\
$T(\mathrm{~K})$ & $298(2)$ & $173(2)$ \\
$D$ calcd $(\mathrm{g}$ cm & -3 & 1.478 \\
$\left.\mu(\mathrm{mm})^{-1}\right)$ & 1.362 & 0.972 \\
$F(000)$ & 0.962 & 4296 \\
No. of rflns. collected & 3952 & 106027 \\
No. of indep. rflns. $/ R_{\text {int }}$ & 49326 & $16505 / 0.0697$ \\
No. of obsd. rflns. $\left[I_{0}>2 \sigma\left(I_{0}\right)\right]$ & $15461 / 0.0573$ & 15369 \\
Data / restraints / parameters & 13671 & $16505 / 2 / 1033$ \\
$R_{I} / w R_{2}\left[I_{0}>2 \sigma\left(I_{0}\right)\right]^{\mathrm{a}}$ & $15461 / 2 / 1009$ & $0.0482 / 0.1099$ \\
$R_{I} / w R_{2}(\text { all data })^{\mathrm{a}}$ & $0.0605 / 0.1249$ & $0.0551 / 0.1146$ \\
$\mathrm{GOF}\left(\text { on } F^{2}\right)^{\mathrm{a}}$ & $0.0605 / 0.1293$ & 1.009 \\
Largest diff. peak and hole $\left(\mathrm{e} \AA^{-3}\right)$ & 0.996 & $0.776 /-0.849$ \\
\hline
\end{tabular}


Figure S1. ORTEP diagram of 1

Thermal ellipsoids are shown at $30 \%$ probability level. $\mathrm{PF}_{6}{ }^{-}$anion and all hydrogen atoms on carbons are omitted for clarity.

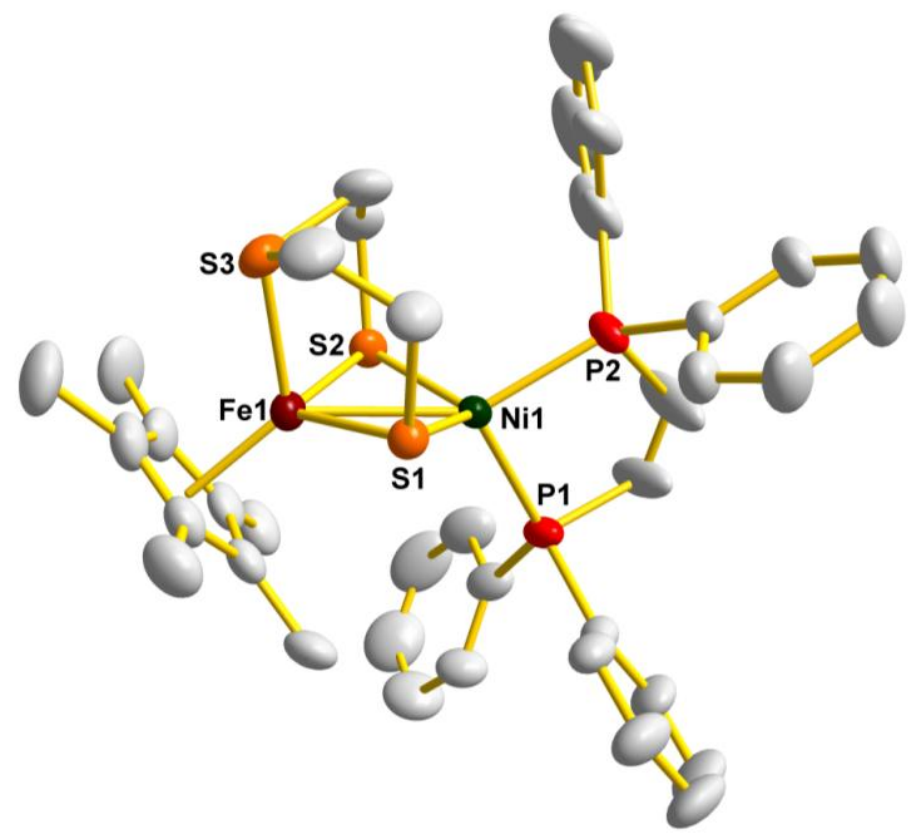

Table S4. Selected bond distances $(\AA)$ and bond angles $\left({ }^{\circ}\right)$ for 1

\begin{tabular}{llll}
\hline Distances $(\AA)$ & & & \\
Fe1-Ni1 & $2.5941(8)$ & Ni1-S1 & $2.1888(13)$ \\
Fe1-S1 & $2.2333(13)$ & Ni1-S2 & $2.1787(13)$ \\
Fe1-S2 & $2.2256(14)$ & Ni1-P1 & $2.2740(13)$ \\
Fe1-S3 & $2.2192(15)$ & Ni1-P2 & $2.1890(14)$ \\
Fe1-Cp*1 & $1.7617(6)$ & & \\
\hline Angles $\left(^{\circ}\right)$ & & & $89.12(6)$ \\
Fe1-S1-Ni1 & $71.83(4)$ & S3-Fe1-S2 & $101.13(5)$ \\
Fe1-S2-Ni1 & $72.16(4)$ & S3-Fe1-Ni1 & $117.97(5)$ \\
Ni1-Fe1-S1 & $53.29(3)$ & S1-Ni1-P1 & $148.33(5)$ \\
Fe1-Ni1-S1 & $54.88(4)$ & Fe1-Ni1-P2 & $110.53(6)$ \\
Ni1-Fe1-S2 & $53.08(4)$ & S2-Ni1-P2 & $122.24(4)$ \\
Fe1-Ni1-S2 & $54.75(4)$ & Fe1-Ni1-P1 & $122.84(6)$ \\
S1-Fe1-S2 & $104.02(5)$ & S2-Ni1-P1 & $118.77(6)$ \\
S1-Ni1-S2 & $107.15(5)$ & S1-Ni1-P2 & $88.83(5)$ \\
S3-Fe1-S1 & $88.33(5)$ & P1-Ni1-P2 & \\
\hline Torsion angles $\left(^{\circ}\right)$ & & & $88.68(4)$ \\
S1-Fe1Fe2-S2 & $159.73(6)$ & Ni1S1S2/Ni1P1P2 & \\
Ni1S1S2/Fe1S1S2 & $27.24(5)$ & & \\
\hline
\end{tabular}


Figure S2. ORTEP diagram of 2

Thermal ellipsoids are shown at $30 \%$ probability level. $\mathrm{PF}_{6}{ }^{-}$anion and all hydrogen atoms on carbons are omitted for clarity.

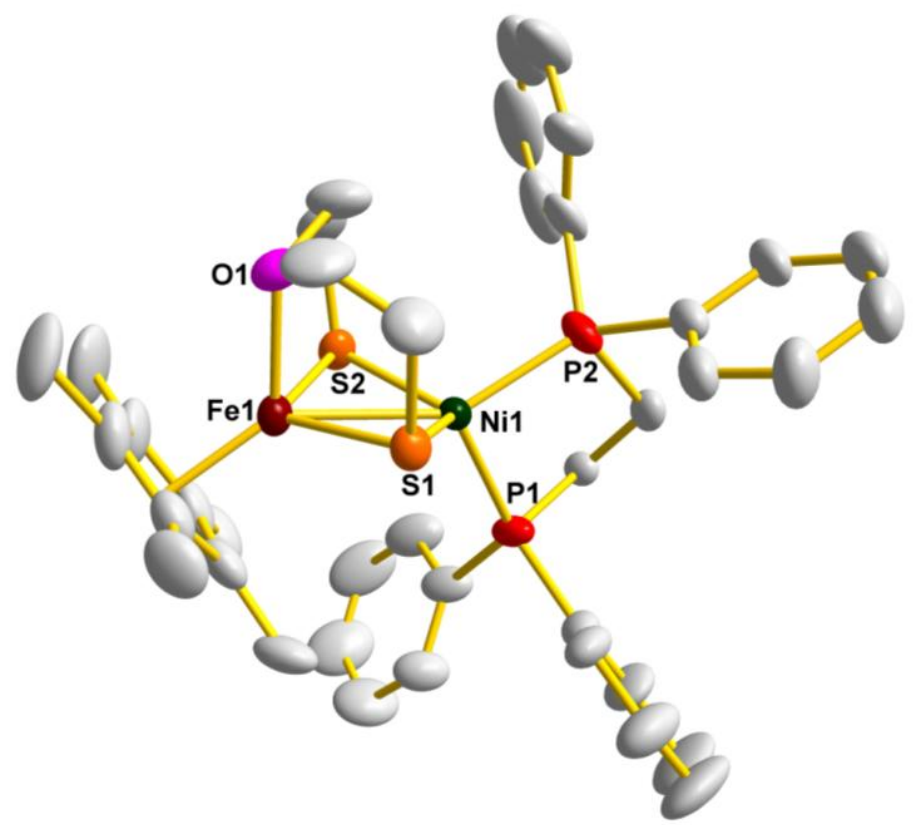

Table S5. Selected bond distances $(\AA)$ and bond angles $\left({ }^{\circ}\right)$ for 2

\begin{tabular}{llll}
\hline Distances $(\AA)$ & & & \\
Fe1-Ni1 & $2.5717(9)$ & Ni1-S1 & $2.1878(13)$ \\
Fe1-S1 & $2.2319(14)$ & Ni1-S2 & $2.1804(13)$ \\
Fe1-S2 & $2.2191(14)$ & Ni1-P1 & $2.2607(14)$ \\
Fe1-O1 & $2.049(4)$ & Ni1-P2 & $2.1759(14)$ \\
Fe1-Cp*1 & $1.7462(7)$ & & \\
\hline Angles $\left(^{\circ}\right)$ & & & $86.22(12)$ \\
Fe1-S1-Ni1 & $71.16(4)$ & O1-Fe1-S2 & $94.42(12)$ \\
Fe1-S2-Ni1 & $71.53(4)$ & O1-Fe1-Ni1 & $116.59(5)$ \\
Ni1-Fe1-S1 & $53.62(3)$ & S1-Ni1-P1 & $147.95(5)$ \\
Fe1-Ni1-S1 & $55.22(4)$ & Fe1-Ni1-P2 & $109.50(6)$ \\
Ni1-Fe1-S2 & $53.53(4)$ & S2-Ni1-P2 & $122.10(4)$ \\
Fe1-Ni1-S2 & $54.93(4)$ & Fe1-Ni1-P1 & $122.30(6)$ \\
S1-Fe1-S2 & $105.24(5)$ & S2-Ni1-P1 & $120.17(6)$ \\
S1-Ni1-S2 & $108.13(5)$ & S1-Ni1-P2 & $89.13(6)$ \\
O1-Fe1-S1 & $84.53(12)$ & P1-Ni1-P2 & \\
\hline Torsion angles $\left(^{\circ}\right)$ & & & $87.53(5)$ \\
S1-Fe1Ni1-S2 & $161.88(6)$ & Ni1S1S2/Ni1P1P2 & \\
Ni1S1S2/Fe1S1S2 & $24.76(6)$ & & \\
\hline
\end{tabular}


Figure S3. ORTEP diagram of 3

Thermal ellipsoids are shown at $30 \%$ probability level. $\mathrm{PF}_{6}{ }^{-}$anion and all hydrogen atoms on carbons are omitted for clarity.

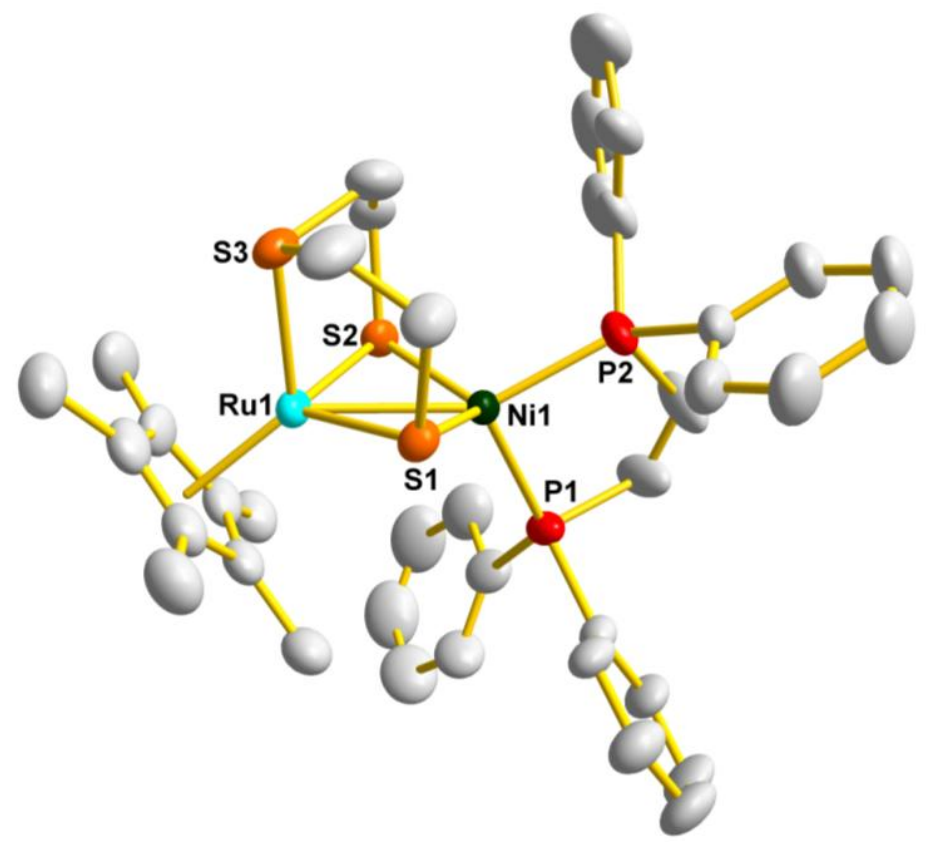

Table S6. Selected bond distances $(\AA)$ and bond angles $\left({ }^{\circ}\right)$ for $\mathbf{3}$

\begin{tabular}{llll}
\hline Distances $(\AA)$ & & & \\
Ru1-Ni1 & $2.6329(9)$ & Ni1-S1 & $2.1918(18)$ \\
Ru1-S1 & $2.2981(17)$ & Ni1-S2 & $2.1798(18)$ \\
Ru1-S2 & $2.2998(19)$ & Ni1-P1 & $2.2839(18)$ \\
Ru1-S3 & $2.3015(18)$ & Ni1-P2 & $2.1718(19)$ \\
Ru1-Cp*1 & $1.8821(5)$ & & \\
\hline Angles $\left(^{\circ}\right)$ & & & $87.13(7)$ \\
Ru1-S1-Ni1 & $71.76(5)$ & S3-Ru1-S2 & $97.93(6)$ \\
Ru1-S2-Ni1 & $71.94(5)$ & S3-Ru1-Ni1 & $117.09(7)$ \\
Ru1-Ni1-S1 & $56.00(5)$ & S1-Ni1-P1 & $110.49(8)$ \\
Ni1-Ru1-S2 & $51.92(4)$ & S2-Ni1-P2 & $119.36(6)$ \\
Ru1-Ni1-S2 & $56.15(5)$ & Ru1-Ni1-P1 & $110.85(8)$ \\
S1-Ru1-S2 & $101.71(6)$ & S2-Ni1-P1 & $118.56(8)$ \\
S1-Ni1-S2 & $109.32(7)$ & S1-Ni1-P2 & $89.21(7)$ \\
S3-Ru1-S1 & $86.19(7)$ & P1-Ni1-P2 & \\
Ni1-Ru1-S1 & $52.24(5)$ & & $89.34(6)$ \\
\hline Torsion angles $\left(^{\circ}\right)$ & & & \\
S1-Ni1Ru1-S2 & $158.89(8)$ & Ni1S1S2/Ni1P1P2 & \\
Ni1S1S2/Ru1S1S2 & $28.46(7)$ & & \\
\hline
\end{tabular}


Figure S4. ORTEP diagram of 4

Thermal ellipsoids are shown at $30 \%$ probability level. $\mathrm{PF}_{6}{ }^{-}$anion and all hydrogen atoms on carbons are omitted for clarity.

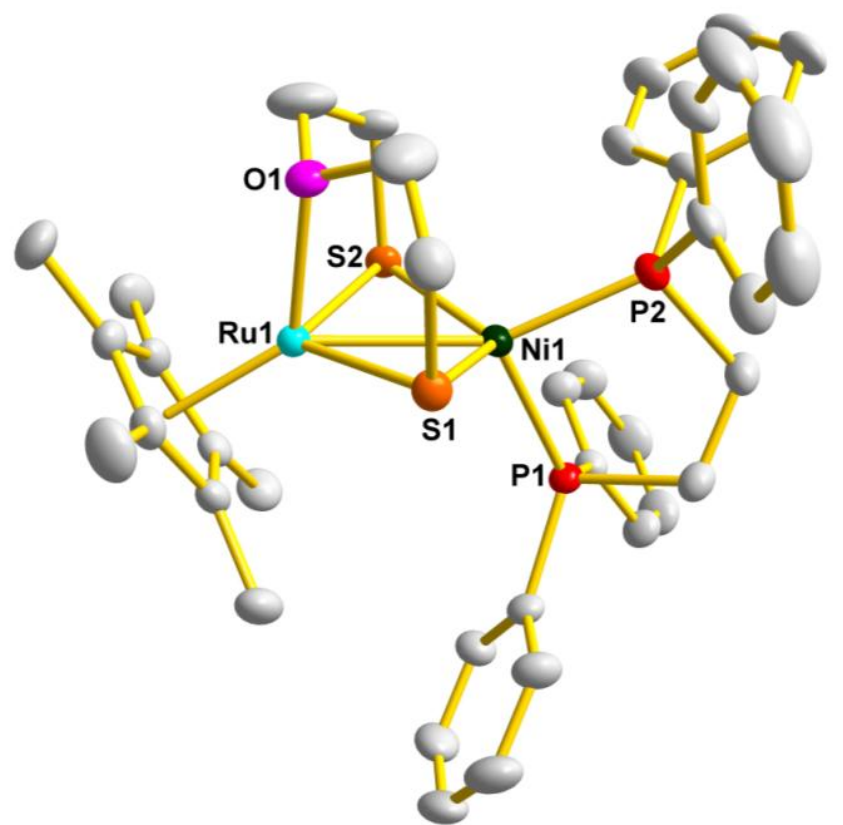

Table S7. Selected bond distances $(\AA)$ and bond angles $\left({ }^{\circ}\right)$ for 4

\begin{tabular}{llll}
\hline Distances $(\AA)$ & & & \\
Ru1-Ni1 & $2.6236(7)$ & Ni1-S1 & $2.1879(15)$ \\
Ru1-S1 & $2.2945(14)$ & Ni1-S2 & $2.1888(15)$ \\
Ru1-S2 & $2.3102(13)$ & Ni1-P1 & $2.1796(15)$ \\
Ru1-O1 & $2.170(4)$ & Ni1-P2 & $2.2796(14)$ \\
Ru1-Cp*1 & $1.8423(4)$ & & \\
\hline Angles $\left(^{\circ}\right)$ & & & $82.27(11)$ \\
Ru1-S1-Ni1 & $71.61(4)$ & O1-Ru1-S2 & $89.13(11)$ \\
Ru1-S2-Ni1 & $71.29(4)$ & O1-Ru1-Ni1 & $107.84(6)$ \\
Ru1-Ni1-S1 & $56.09(4)$ & S1-Ni1-P1 & $119.00(6)$ \\
Ni1-Ru1-S2 & $52.20(4)$ & S2-Ni1-P2 & $119.91(4)$ \\
Ru1-Ni1-S2 & $56.51(4)$ & Ru1-Ni1-P1 & $117.57(6)$ \\
S1-Ru1-S2 & $102.84(5)$ & S2-Ni1-P1 & $110.14(6)$ \\
S1-Ni1-S2 & $110.67(6)$ & S1-Ni1-P2 & $89.72(6)$ \\
O1-Ru1-S1 & $82.99(12)$ & P1-Ni1-P2 & \\
Ni1-Ru1-S1 & $52.31(4)$ & & $89.36(4)$ \\
\hline Torsion angles $\left(^{\circ}\right)$ & & & \\
S1-Ni1Ru1-S2 & $162.69(6)$ & Ni1S1S2/Ni1P1P2 & \\
Ni1S1S2/Ru1S1S2 & $23.71(6)$ & & \\
\hline
\end{tabular}


Figure S5. ORTEP diagram of 5

Thermal ellipsoids are shown at $30 \%$ probability level. $\mathrm{PF}_{6}{ }^{-}$anion and all hydrogen atoms on carbons are omitted for clarity.

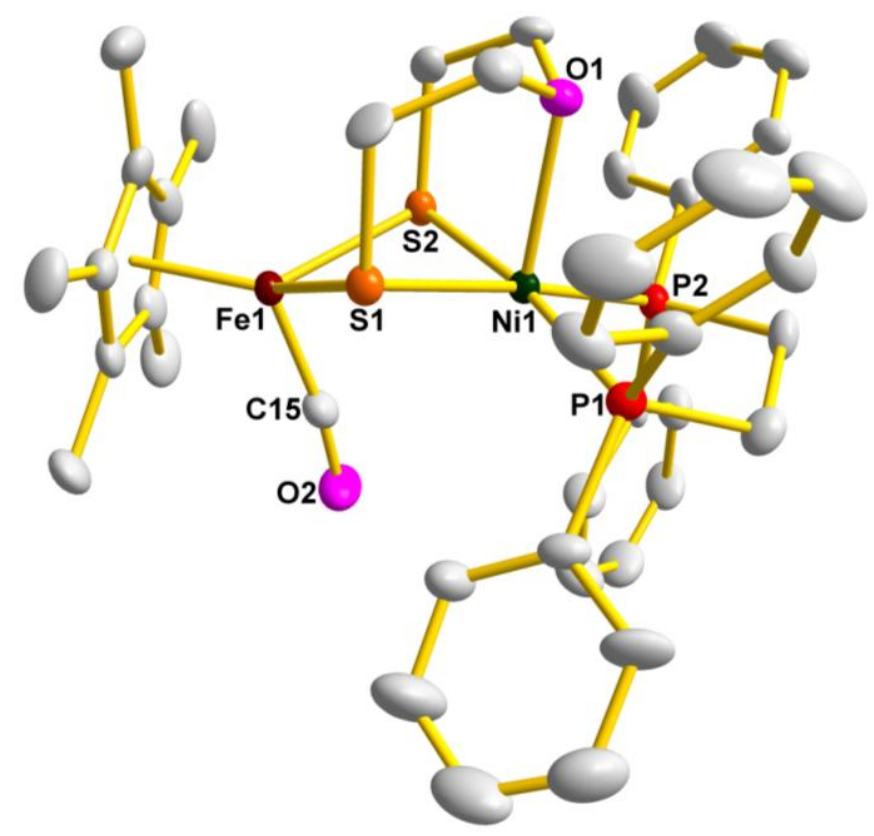

Table S8. Selected bond distances $(\AA)$ and bond angles $\left({ }^{\circ}\right)$ for $\mathbf{5}$

\begin{tabular}{llllll}
\hline & Molecule 1 & Molecule 2 & & Molecule 1 & Molecule 2 \\
\hline Distances $(\AA)$ & & & & & \\
Fe1 $\cdots$ Ni1 & $3.1648(10)$ & $3.1618(11)$ & Ni1-S1 & $2.2508(16)$ & $2.3884(16)$ \\
Fe1-S1 & $2.3085(17)$ & $2.3080(18)$ & Ni1-S2 & $2.2371(15)$ & $2.2465(16)$ \\
Fe1-S2 & $2.2994(16)$ & $2.2961(17)$ & Ni1-P1 & $2.1847(16)$ & $2.1805(16)$ \\
Ni1-O1 & $2.345(4)$ & $2.345(4)$ & Ni1-P2 & $2.1786(15)$ & $2.1795(15)$ \\
Fe1-C15 & $1.737(7)$ & $1.761(8)$ & C15-O2 & $1.147(8)$ & $1.133(8)$ \\
Fe1-Cp*1 & $1.7319(7)$ & $1.7353(9)$ & & & \\
\hline Angles $\left(^{\circ}\right)$ & & & & & \\
Fe1-S1-Ni1 & $88.24(5)$ & $88.11(6)$ & O1-Ni1-S2 & $84.83(12)$ & $84.81(12)$ \\
Fe1-S2-Ni1 & $88.14(6)$ & $88.21(6)$ & O1-Fe1-Ni1 & $34.45(7)$ & $31.75(7)$ \\
Ni1-Fe1-S1 & $44.96(4)$ & $45.04(4)$ & Fe1-C15-O2 & $171.0(6)$ & $170.1(6)$ \\
Fe1-Ni1-S1 & $46.81(4)$ & $46.85(4)$ & S1-Ni1-P1 & $92.55(6)$ & $92.74(6)$ \\
Ni1-Fe1-S2 & $45.30(4)$ & $45.25(4)$ & S2-Ni1-P2 & $92.94(6)$ & $92.90(6)$ \\
Fe1-Ni1-S2 & $46.57(4)$ & $46.54(4)$ & Fe1-Ni1-P1 & $127.84(5)$ & $128.60(5)$ \\
S1-Fe1-S2 & $83.53(6)$ & $83.76(6)$ & S2-Ni1-P1 & $170.96(7)$ & $171.63(7)$ \\
S1-Ni1-S2 & $86.29(6)$ & $86.52(6)$ & S1-Ni1-P2 & $174.57(7)$ & $174.70(7)$ \\
O1-Ni1-S1 & $86.27(11)$ & $85.59(12)$ & P1-Ni1-P2 & $87.37(6)$ & $87.07(6)$ \\
\hline Torsion angles $\left(^{\circ}\right)$ & & & & & \\
S1-Ni1Fe1-S2 & $140.07(8)$ & $140.69(9)$ & Ni1S1S2/Ni1P1P2 & $10.28(5)$ & $9.67(5)$ \\
Ni1S1S2/Fe1S1S2 & $38.84(5)$ & $38.32(7)$ & & & \\
\hline
\end{tabular}


Figure S6. ORTEP diagram of 6

Thermal ellipsoids are shown at $30 \%$ probability level. $\mathrm{PF}_{6}{ }^{-}$anion and all hydrogen atoms on carbons are omitted for clarity.

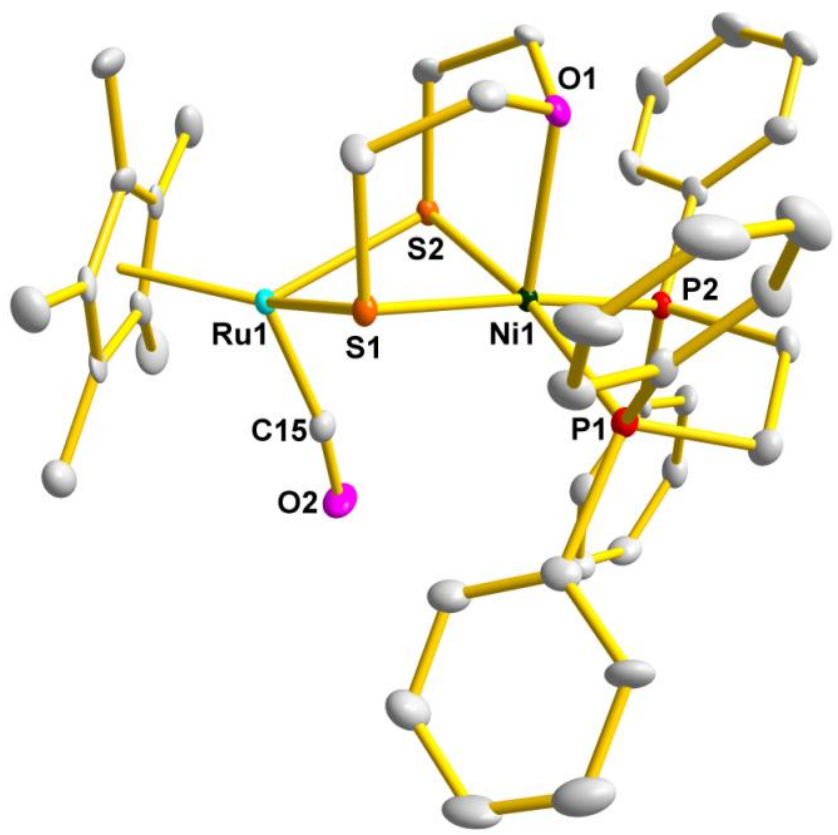

Table S9. Selected bond distances $(\AA)$ and bond angles $\left({ }^{\circ}\right)$ for $\mathbf{6}$

\begin{tabular}{|c|c|c|c|c|c|}
\hline & Molecule 1 & Molecule 2 & & Molecule 1 & Molecule 2 \\
\hline \multicolumn{6}{|l|}{ Distances $(\AA)$} \\
\hline Ru1 $\cdots \mathrm{Ni} 1$ & $3.2790(9)$ & $3.2864(8)$ & $\mathrm{Ni1}-\mathrm{S} 1$ & $2.2463(17)$ & $2.2400(16)$ \\
\hline $\mathrm{Ru} 1-\mathrm{S} 1$ & $2.4235(17)$ & $2.4237(15)$ & Ni1-S2 & $2.2440(17)$ & $2.2533(17)$ \\
\hline $\mathrm{Ru} 1-\mathrm{S} 2$ & $2.4092(16)$ & $2.4092(15)$ & Ni1-P1 & $2.1680(18)$ & $2.1746(18)$ \\
\hline Ni1-O1 & $2.357(4)$ & $2.361(4)$ & $\mathrm{Ni1}-\mathrm{P} 2$ & $2.1658(17)$ & $2.1651(17)$ \\
\hline Ru1-C15 & $1.854(7)$ & $1.857(7)$ & $\mathrm{C} 15-\mathrm{O} 2$ & $1.155(8)$ & $1.146(8)$ \\
\hline Ru1-Cp*1 & $1.8593(5)$ & $1.8591(4)$ & & & \\
\hline \multicolumn{6}{|l|}{ Angles $\left({ }^{\circ}\right)$} \\
\hline $\mathrm{Ru} 1-\mathrm{S} 1-\mathrm{Ni} 1$ & $89.12(6)$ & $89.51(6)$ & $\mathrm{O} 1-\mathrm{Ni1}-\mathrm{S} 2$ & $85.14(12)$ & $84.84(12)$ \\
\hline $\mathrm{Ru} 1-\mathrm{S} 2-\mathrm{Ni} 1$ & $89.53(6)$ & $89.57(6)$ & O1-Ru1-Ni1 & $30.97(7)$ & $30.90(5)$ \\
\hline Ni1-Ru1-S1 & $43.24(4)$ & $42.97(4)$ & $\mathrm{Ru} 1-\mathrm{C} 15-\mathrm{O} 2$ & $168.6(6)$ & $168.6(6)$ \\
\hline Ru1-Ni1-S1 & $47.65(4)$ & $47.52(4)$ & $\mathrm{S} 1-\mathrm{Ni1}-\mathrm{P} 1$ & $92.05(7)$ & $91.49(6)$ \\
\hline $\mathrm{Ni1}-\mathrm{Ru} 1-\mathrm{S} 2$ & $43.18(4)$ & $43.29(4)$ & $\mathrm{S} 2-\mathrm{Ni1}-\mathrm{P} 2$ & $92.07(6)$ & $92.48(6)$ \\
\hline $\mathrm{Ru} 1-\mathrm{Ni1}-\mathrm{S} 2$ & $47.28(4)$ & $47.14(4)$ & $\mathrm{Ru} 1-\mathrm{Ni1}-\mathrm{P} 1$ & $128.04(5)$ & $127.01(5)$ \\
\hline $\mathrm{S} 1-\mathrm{Ru} 1-\mathrm{S} 2$ & $80.34(5)$ & $80.20(5)$ & S2-Ni1-P1 & $170.96(7)$ & $169.98(7)$ \\
\hline $\mathrm{S} 1-\mathrm{Ni1}-\mathrm{S} 2$ & $87.93(6)$ & $87.71(6)$ & $\mathrm{S} 1-\mathrm{Ni1}-\mathrm{P} 2$ & $173.79(7)$ & $173.81(7)$ \\
\hline $\mathrm{O} 1-\mathrm{Ni1}-\mathrm{S} 1$ & $85.44(12)$ & $85.82(11)$ & $\mathrm{P} 1-\mathrm{Ni1}-\mathrm{P} 2$ & $86.97(6)$ & $87.25(6)$ \\
\hline \multicolumn{6}{|l|}{ Torsion angles $\left({ }^{\circ}\right)$} \\
\hline S1-Ni1Ru1-S2 & $140.82(8)$ & $140.87(8)$ & Ni1S1S2/Ni1P1P2 & $10.73(5)$ & $11.54(6)$ \\
\hline Ni1S1S2/Ru1S1S2 & $37.55(6)$ & $37.38(5)$ & & & \\
\hline
\end{tabular}


Figure S7. ESI-HRMS of 1 in $\mathrm{CH}_{2} \mathrm{Cl}_{2}$

(a) The signal at $\mathrm{m} / \mathrm{z}=799.1027$ corresponds to $\left[1-\mathrm{PF}_{6}\right]^{+}$. (b) Calculated isotopic distribution for $\left[1-\mathrm{PF}_{6}\right]^{+}$(upper) and the amplifying experimental diagram for $\left[1-\mathrm{PF}_{6}\right]^{+}$ (bottom).

(a)

(b)

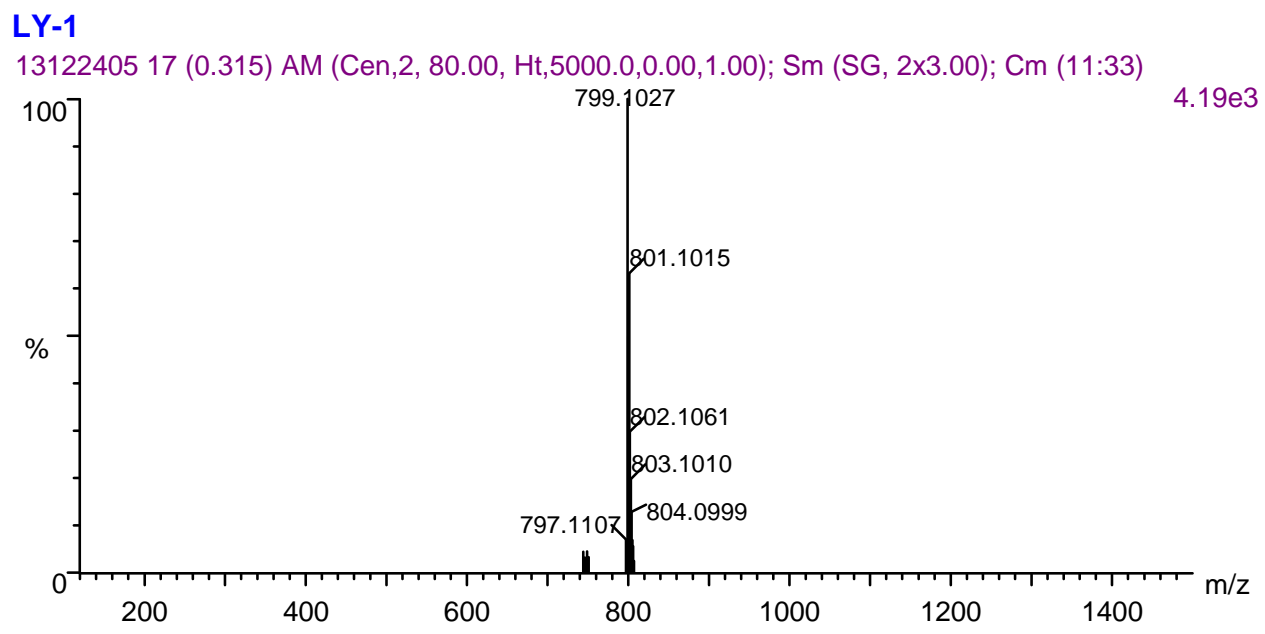

\section{LY-1}

$13122405(0.019)$ Is $(1.00,1.00) \mathrm{C} 40 \mathrm{H} 47 \mathrm{P} 2 \mathrm{~S} 3 \mathrm{~F} \epsilon$

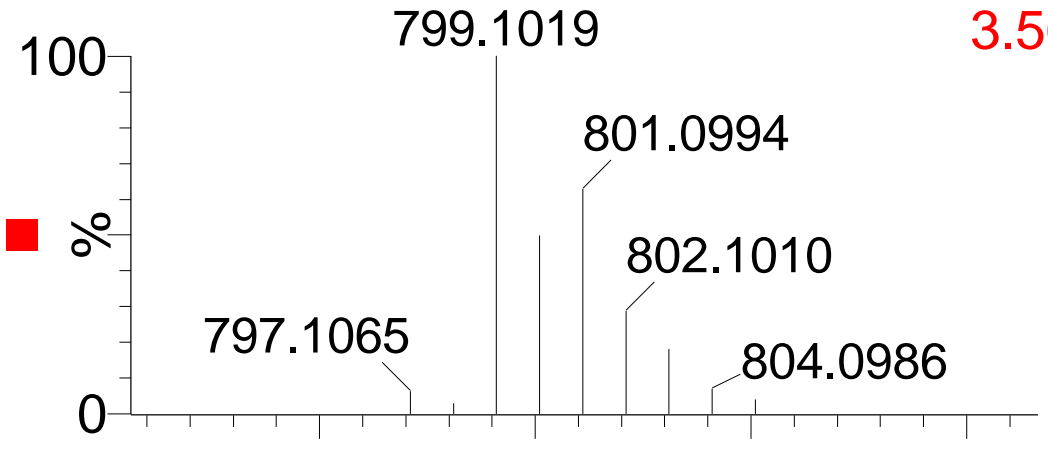

1312240517 (0.315) AM (Cen,2, 80.00, Ht,5000.

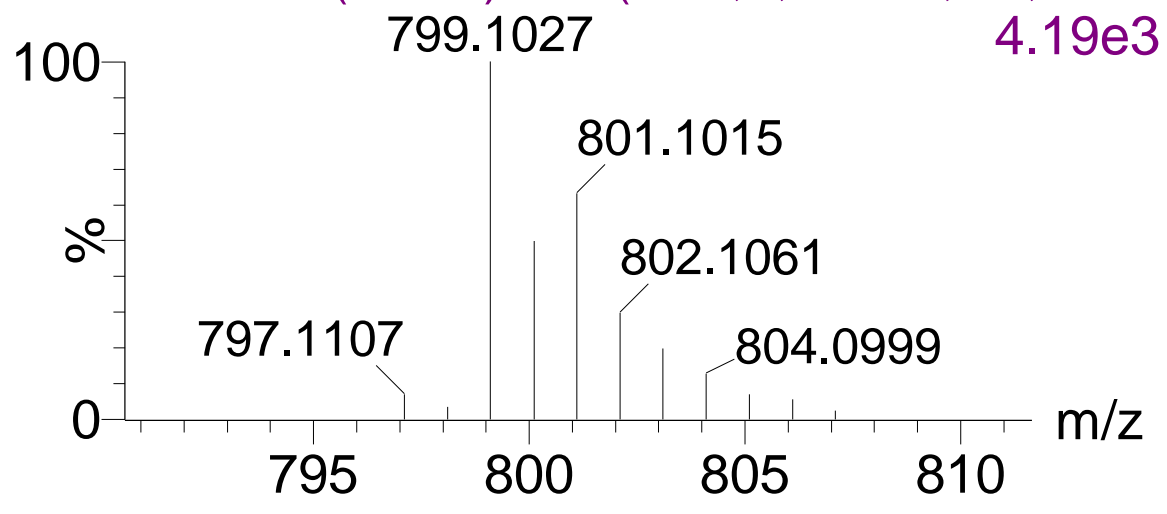


Figure S8. ESI-HRMS of 2 in $\mathrm{CH}_{2} \mathrm{Cl}_{2}$

(a) The signal at $\mathrm{m} / \mathrm{z}=783.1243$ corresponds to $\left[2-\mathrm{PF}_{6}\right]^{+}$. (b) Calculated isotopic distribution for $\left[2-\mathrm{PF}_{6}\right]^{+}$(upper) and the amplifying experimental diagram for $\left[2-\mathrm{PF}_{6}\right]^{+}$ (bottom).

(a)

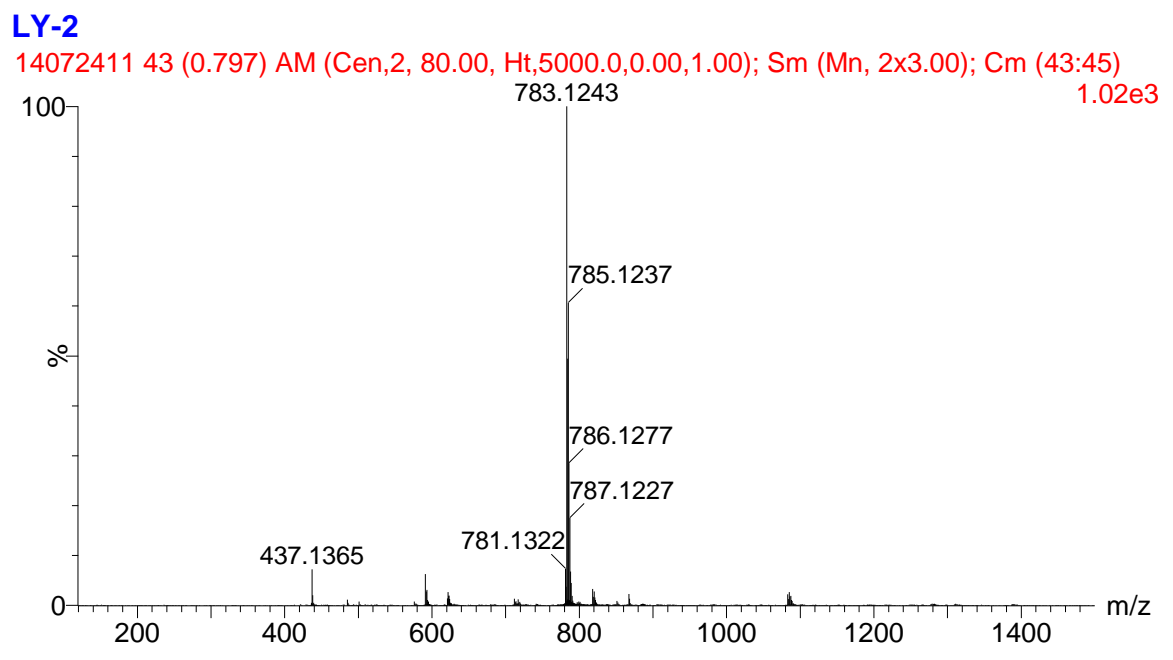

(b)

\section{LY-2}

$14072411(0.019)$ Is $(1.00,1.00) \mathrm{C} 40 \mathrm{H} 47 \mathrm{OP} 2 \mathrm{~S} 2 \mathrm{FeNi}$

100

783.1248

$3.73 \mathrm{e} 12$

785.1224

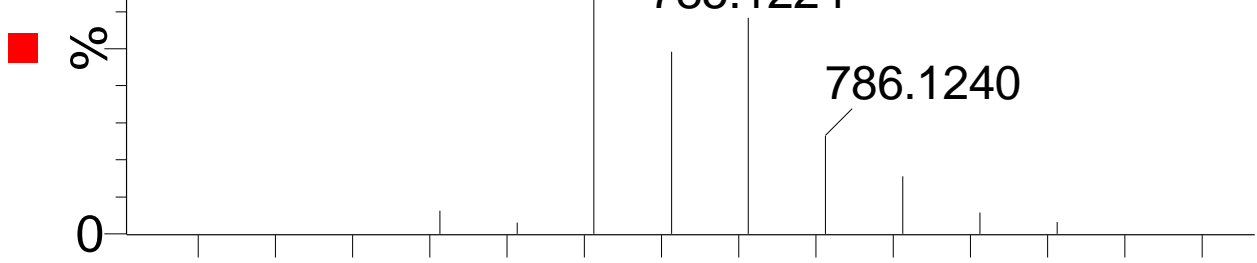

$\begin{array}{rr}1407241143(0.797) & \text { AM (Cen,2, 80.00, Ht,5000.0,0.00,1.00' } \\ 783.1243 & 1.02 \mathrm{e} 3\end{array}$

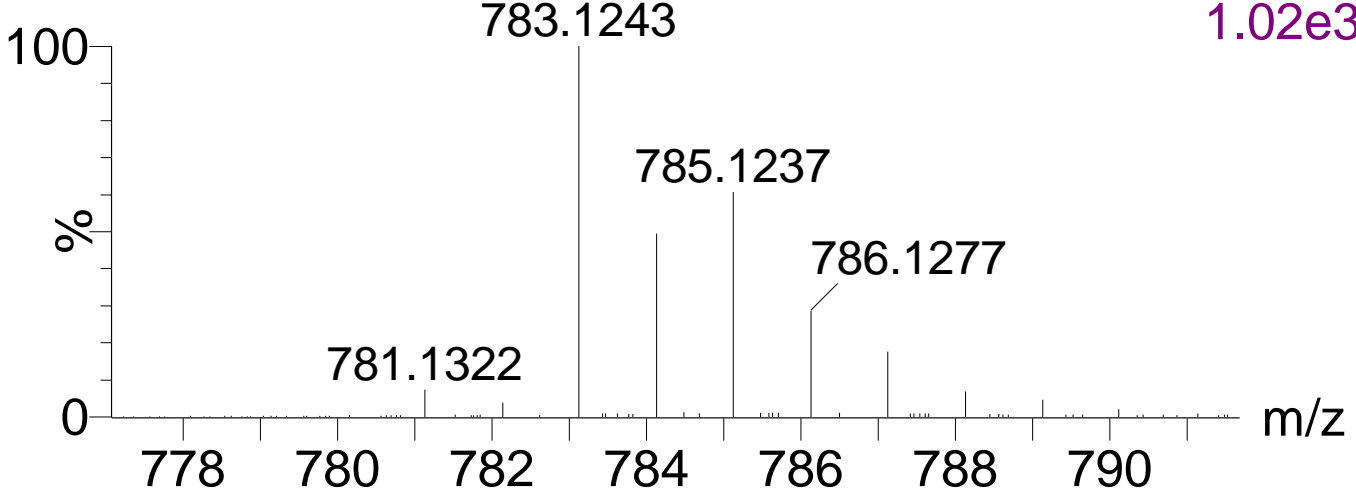


Figure S9. ESI-HRMS of 3 in $\mathrm{CH}_{2} \mathrm{Cl}_{2}$

(a) The signal at $\mathrm{m} / \mathrm{z}=845.0715$ corresponds to $\left[3-\mathrm{PF}_{6}\right]^{+}$(b) Calculated isotopic distribution for $\left[3-\mathrm{PF}_{6}\right]^{+}$(upper) and the amplifying experimental diagram for $\left[3-\mathrm{PF}_{6}\right]^{+}$ (bottom).

(a)

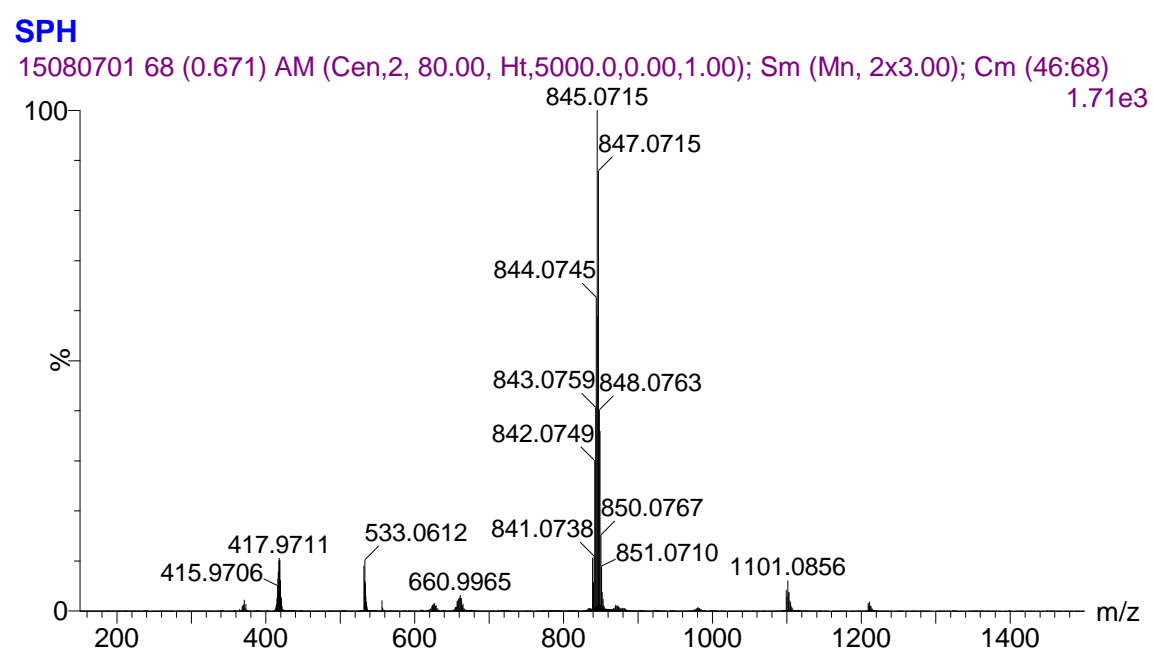

(b)

\section{SPH}

15080701 (0.010) Is $(1.00,1.00) \mathrm{C} 40 \mathrm{H} 47 \mathrm{P} 2 \mathrm{~S} 3 \mathrm{NiRl}$
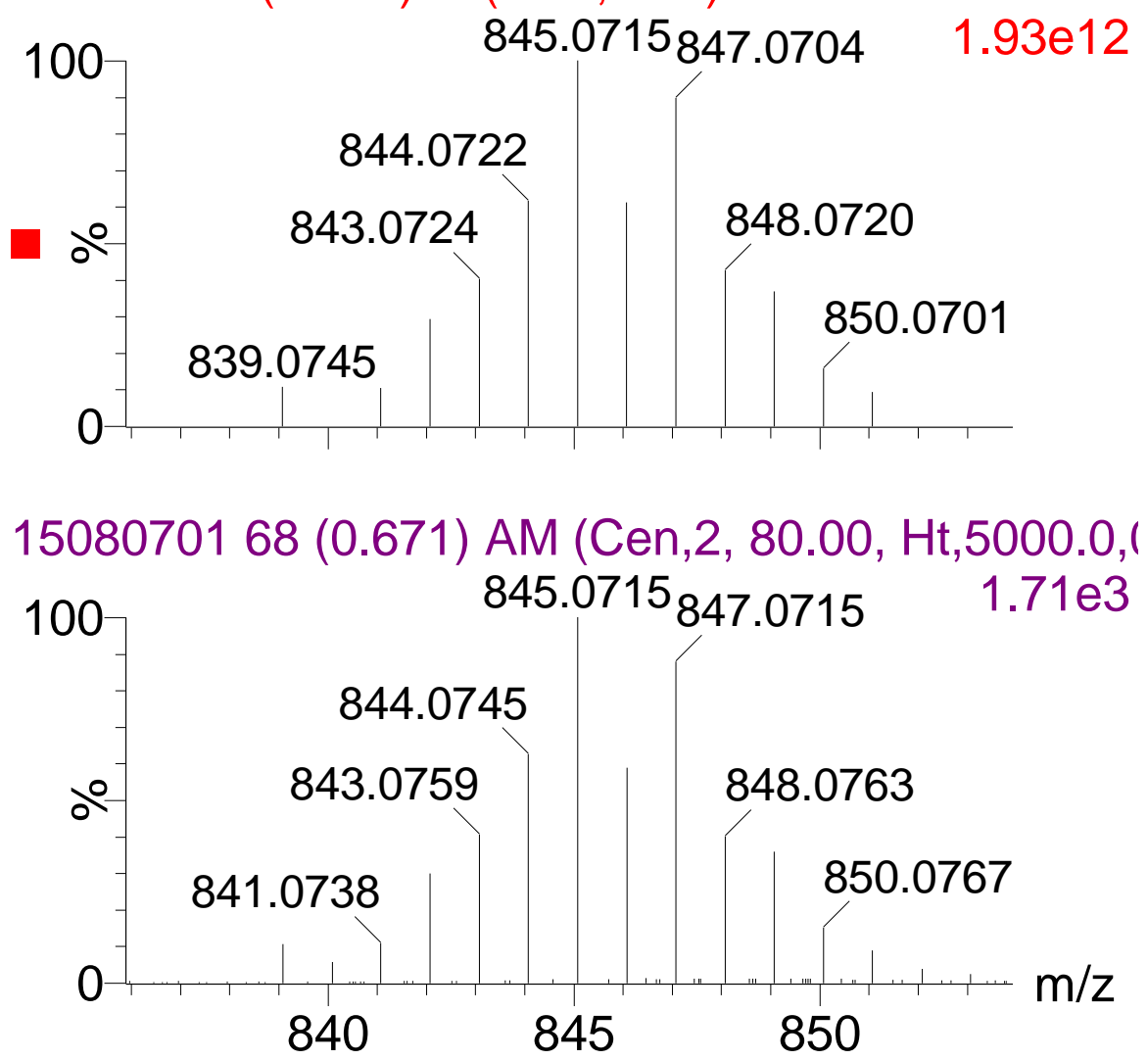
Figure S10. ESI-HRMS of 4 in $\mathrm{CH}_{2} \mathrm{Cl}_{2}$

(a) The signal at $\mathrm{m} / \mathrm{z}=829.0923$ corresponds to $\left[4-\mathrm{PF}_{6}\right]^{+}$(b) Calculated isotopic distribution for $\left[4-\mathrm{PF}_{6}\right]^{+}$(upper) and the amplifying experimental diagram for $\left[4-\mathrm{PF}_{6}\right]^{+}$ (bottom).

(a)

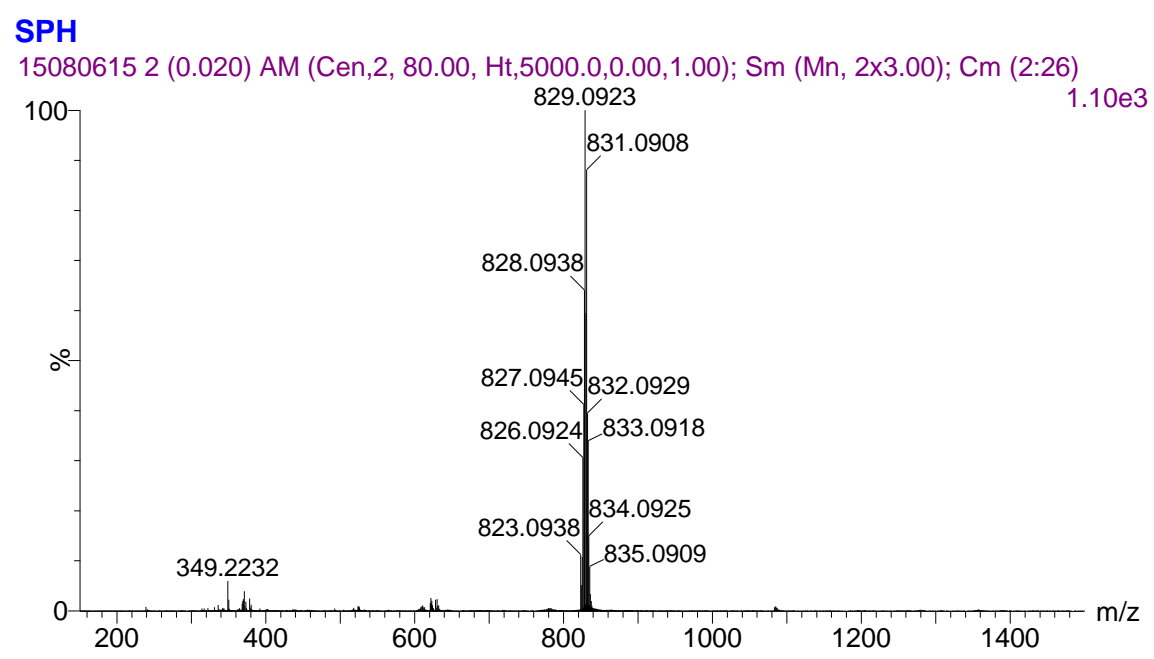

(b)

\section{SPH}

15080615 (0.010) Is $(1.00,1.00) \mathrm{C} 40 \mathrm{H} 47 \mathrm{OP} 2 \mathrm{~S} 2 \mathrm{Ni}$

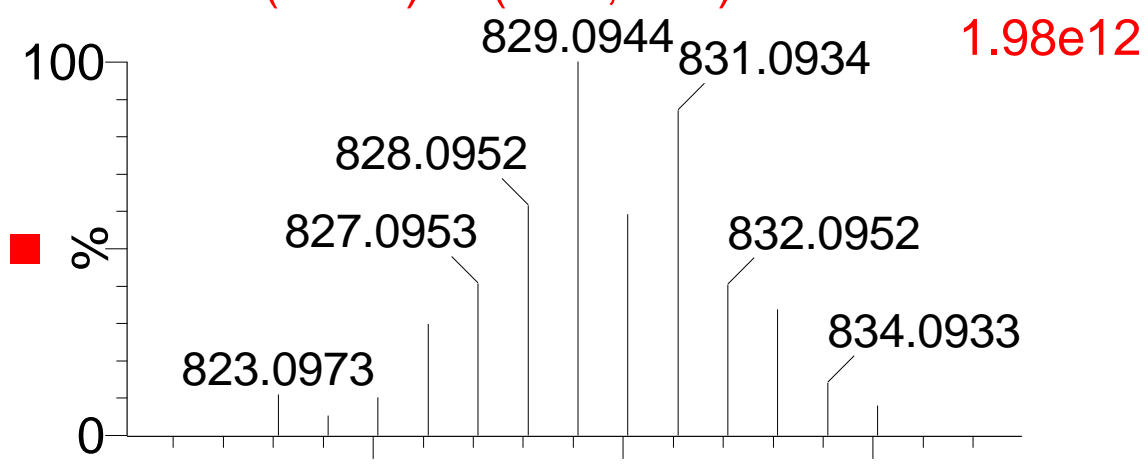

150806152 (0.020) AM (Cen,2, 80.00, Ht,5000.0,0

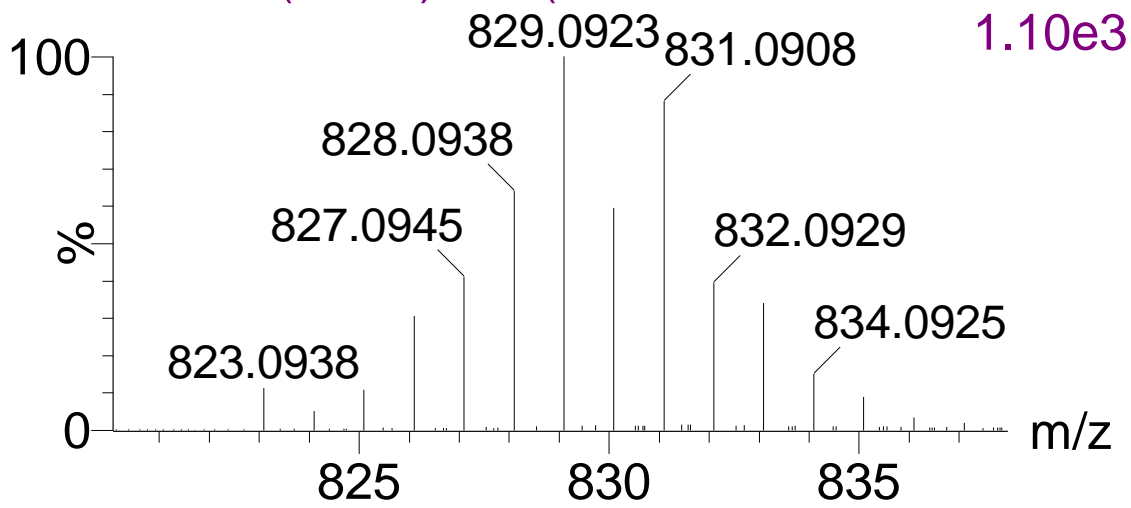


Figure S11. ESI-HRMS of 5 in $\mathrm{CH}_{2} \mathrm{Cl}_{2}$

(a) The signal at $\mathrm{m} / \mathrm{z}=811.1184$ corresponds to $\left[5-\mathrm{PF}_{6}\right]^{+}$(b) Calculated isotopic distribution for $\left[5-\mathrm{PF}_{6}\right]^{+}$(upper) and the amplifying experimental diagram for $\left[5-\mathrm{PF}_{6}\right]^{+}$ (bottom).

(a)

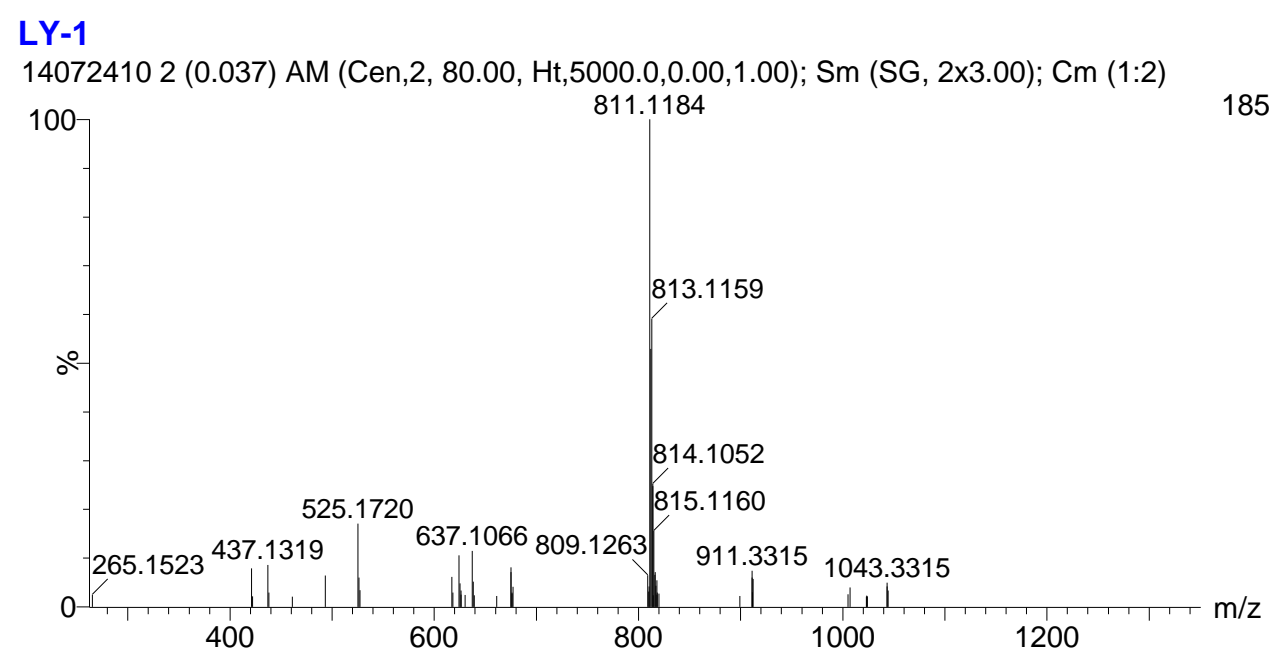

(b)

LY-1

$14072410 \quad(0.019)$ Is $(1.00,1.00) \mathrm{C} 41 \mathrm{H} 47 \mathrm{O} 2 \mathrm{P} 2 \mathrm{~S} 2 \mathrm{FeNi}$

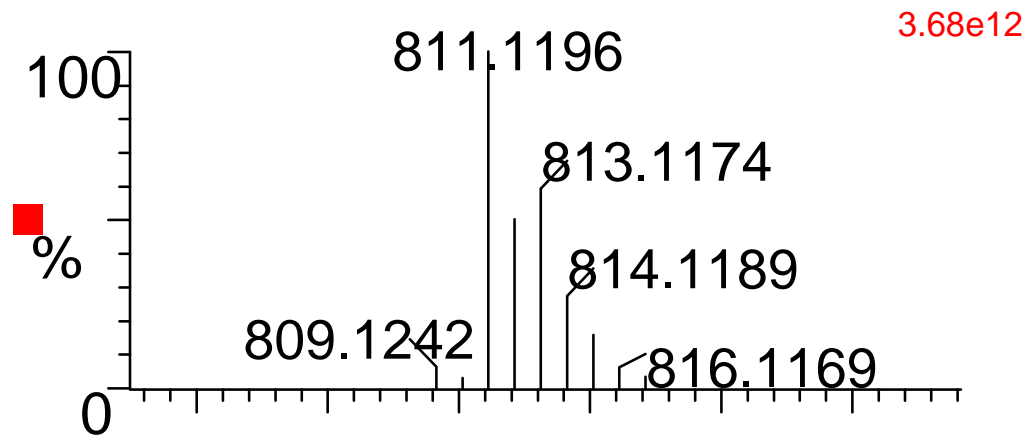

140724102 (0.037) AM (Cen,2, 80.00, Ht,5000.0,0.00,1.00); Sm (SG, 2×3.00); Cm (1:2)

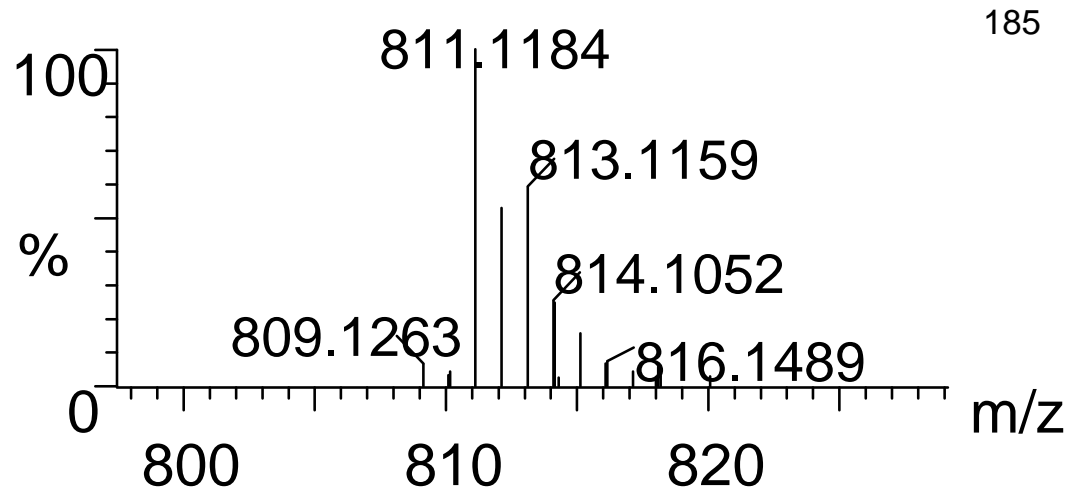


Figure S12. ESI-HRMS of 6 in $\mathrm{CH}_{2} \mathrm{Cl}_{2}$

(a) The signal at $\mathrm{m} / \mathrm{z}=857.0716$ corresponds to $\left[6-\mathrm{PF}_{6}\right]^{+}$(b) Calculated isotopic distribution for $\left[6-\mathrm{PF}_{6}\right]^{+}$(upper) and the amplifying experimental diagram for $\left[6-\mathrm{PF}_{6}\right]^{+}$ (bottom).

(a)

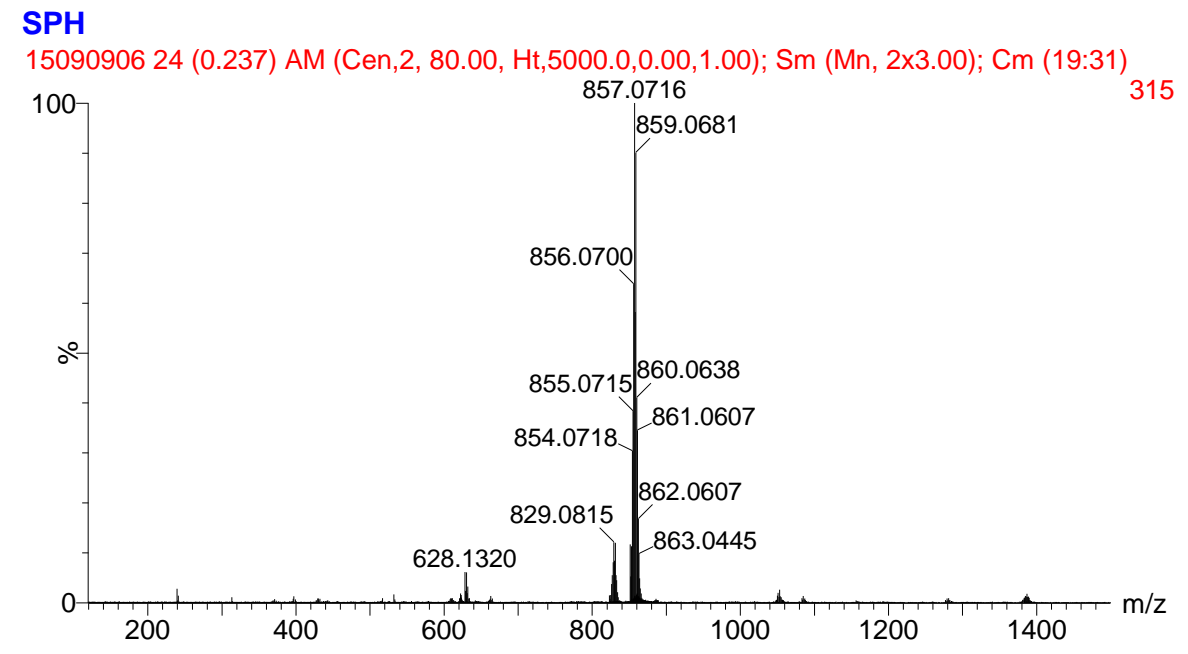

(b)

\section{SPH}

15090906 (0.010) Is $(1.00,1.00) \mathrm{C} 41 \mathrm{H} 47 \mathrm{O} 2 \mathrm{P} 2 \mathrm{~S} 2 \mathrm{~F}$

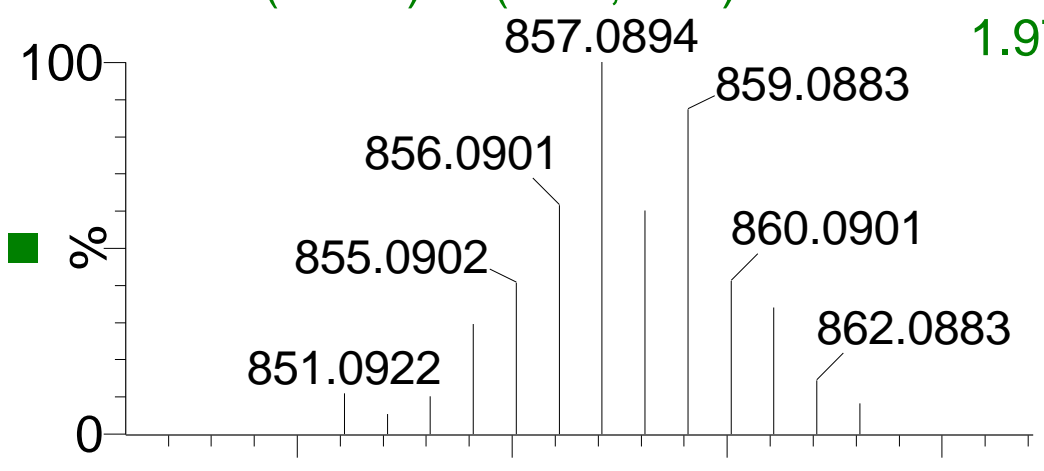

1509090624 (0.237) AM (Cen,2, 80.00, Ht,5000.0,।

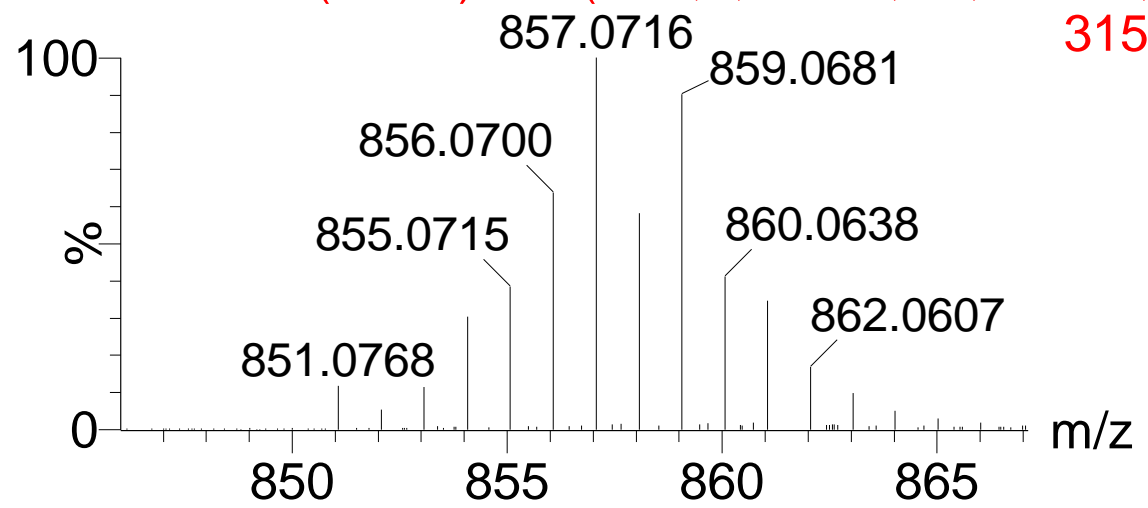


Figure S13. The ${ }^{1} \mathrm{H}$ NMR spectrum of 1 in $\mathrm{CD}_{2} \mathrm{Cl}_{2}$

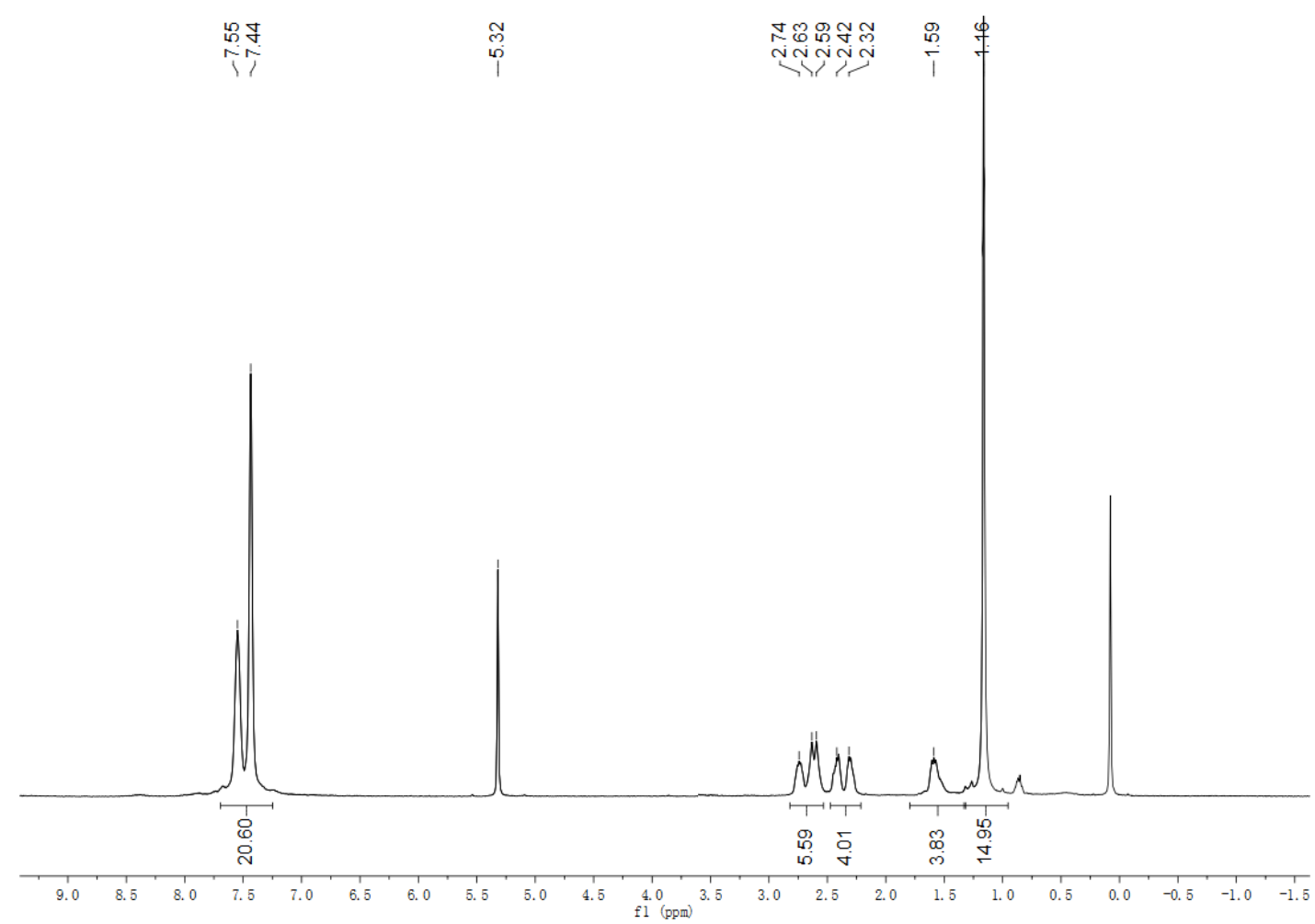

Figure S14. The ${ }^{1} \mathrm{H}$ NMR spectrum of 2 in $\mathrm{CD}_{2} \mathrm{Cl}_{2}$

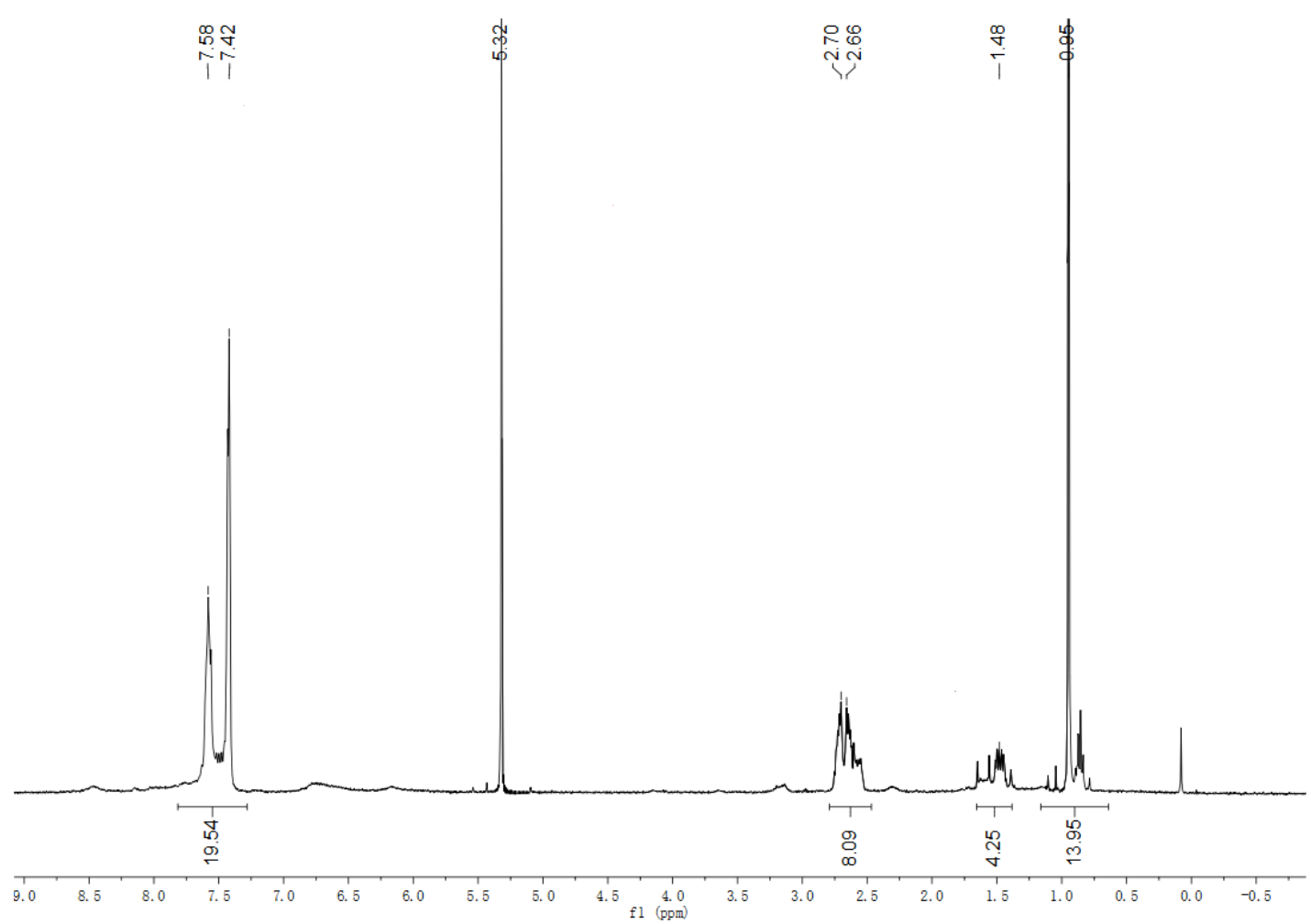


Figure S15. The ${ }^{1} \mathrm{H}$ NMR spectrum of $\mathbf{3}$ in $\mathrm{CD}_{2} \mathrm{Cl}_{2}$

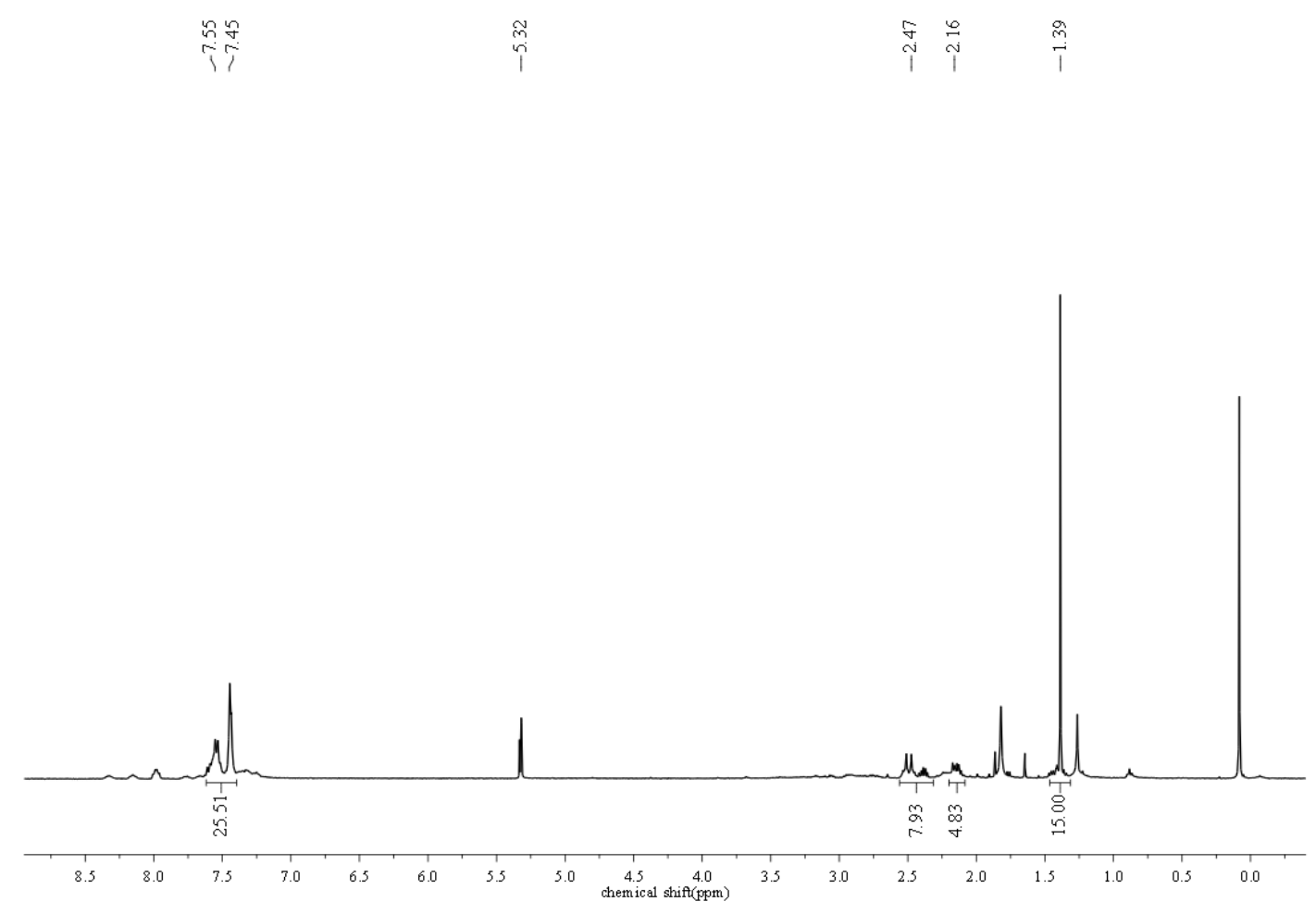

Figure S16. The ${ }^{1} \mathrm{H}$ NMR spectrum of 4 in $\mathrm{CD}_{2} \mathrm{Cl}_{2}$

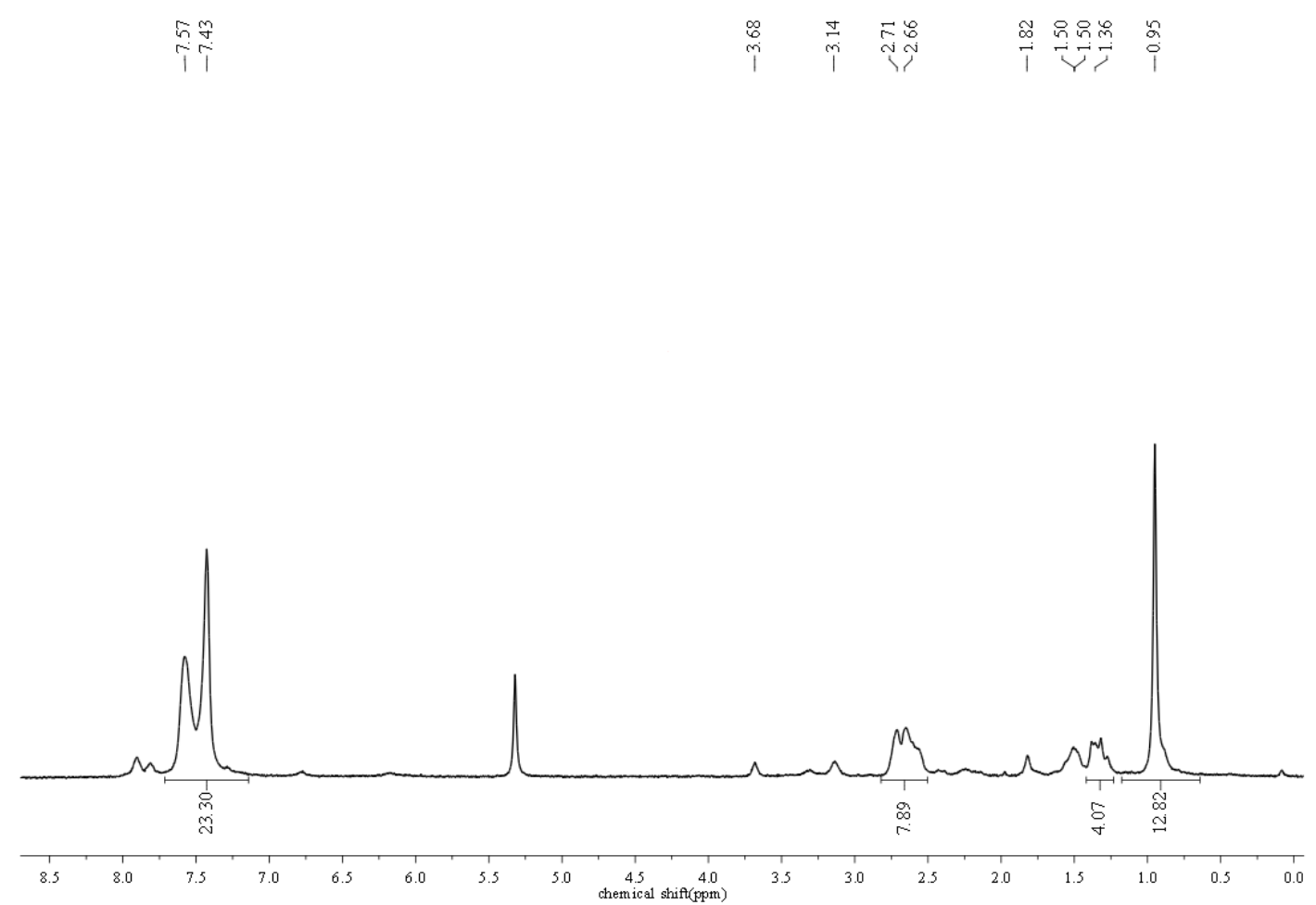


Figure S17. The ${ }^{1} \mathrm{H}$ NMR spectrum of 5 in $\mathrm{CD}_{2} \mathrm{Cl}_{2}$

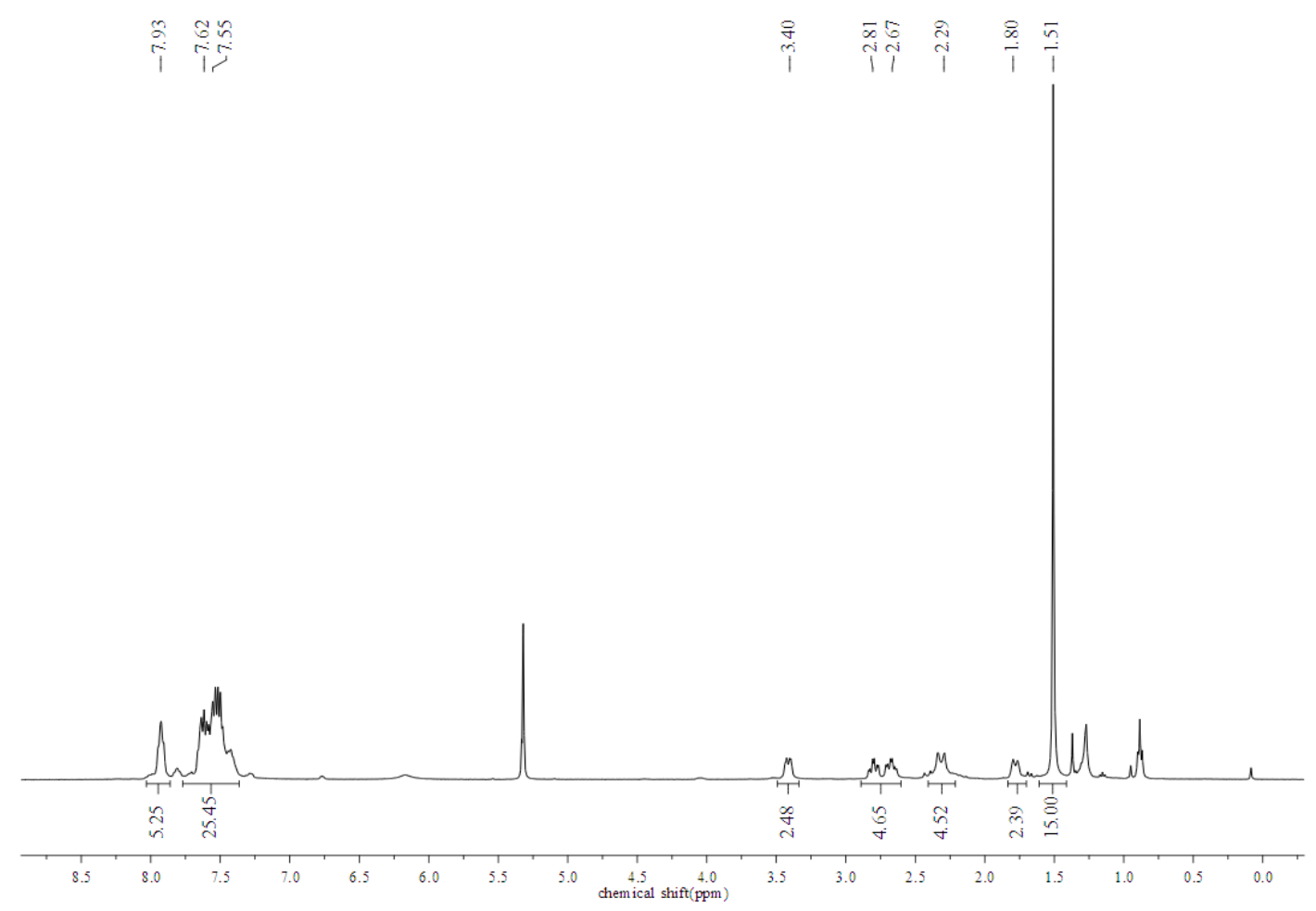

Figure S18. The ${ }^{1} \mathrm{H}$ NMR spectrum of $\mathbf{6}$ in $\mathrm{CD}_{2} \mathrm{Cl}_{2}$

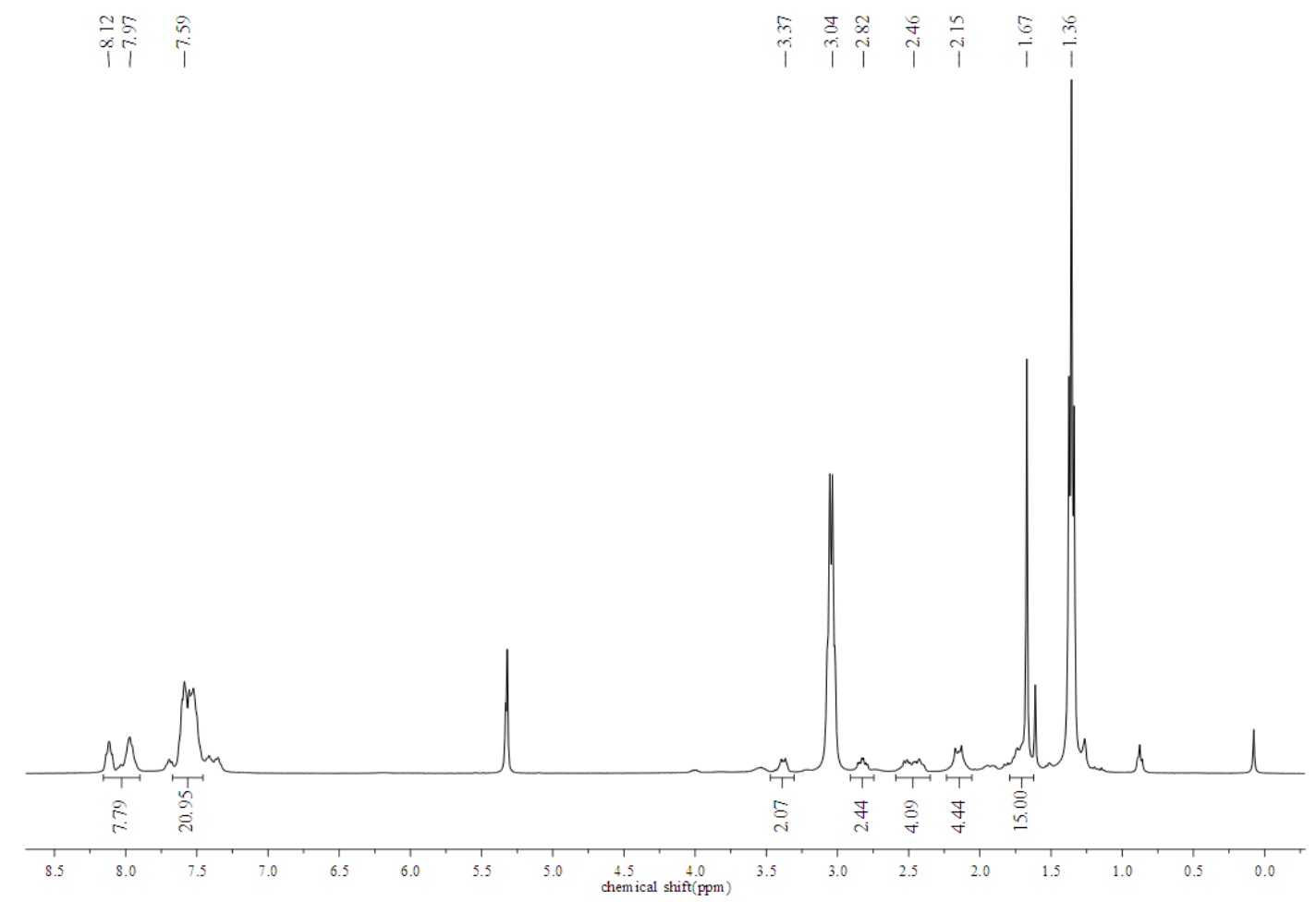

S21 / S31 
Figure S19. The IR (film) spectrum of 1

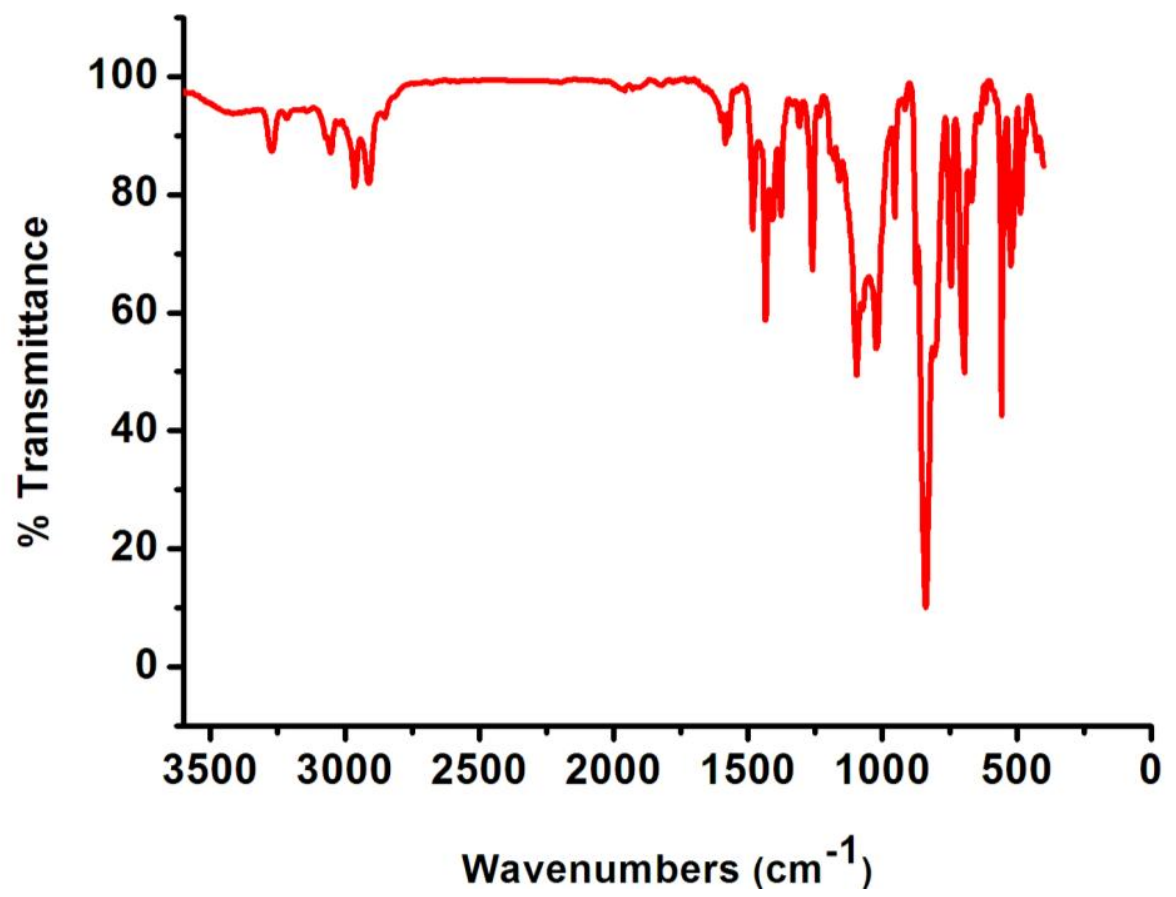

Figure S20. The IR (film) spectrum of 2

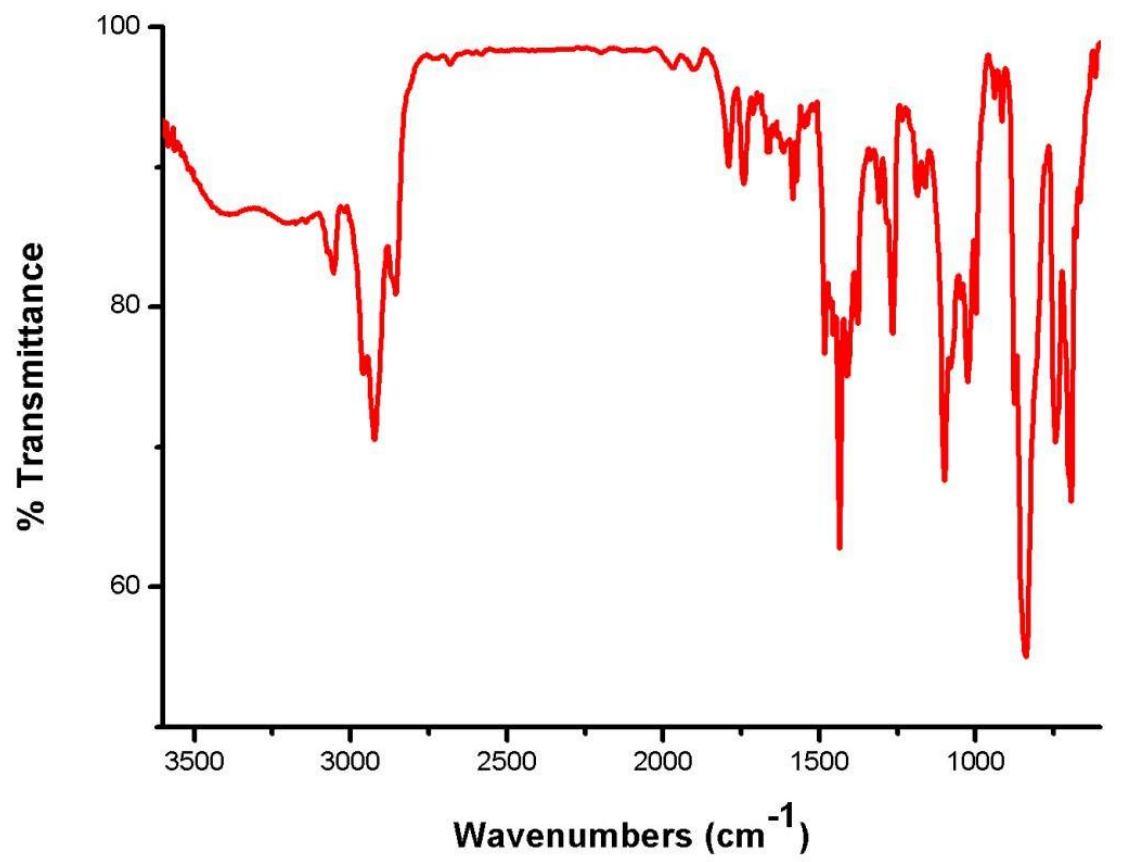


Figure S21. The IR (KBr) spectrum of 3

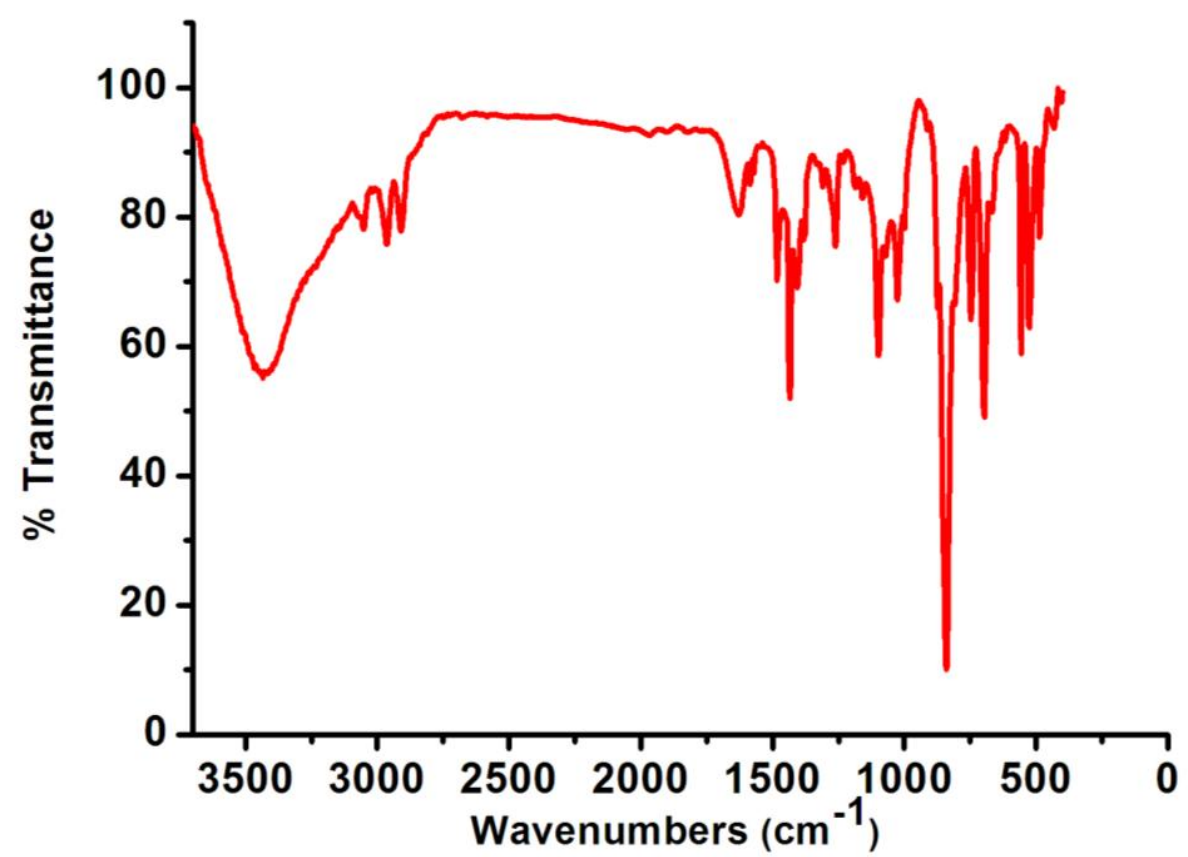

Figure S22. The IR (KBr) spectrum of 4

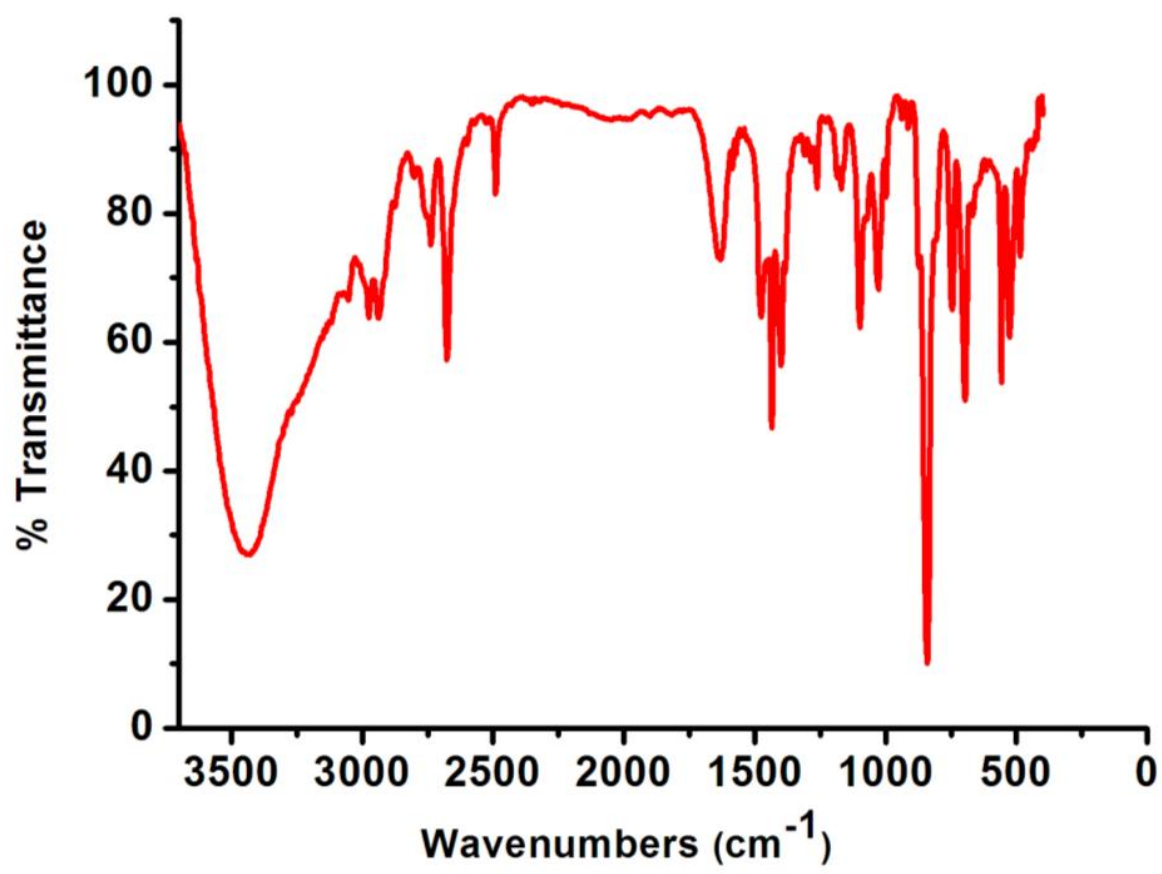


Figure S23. The IR (film) spectrum of 5

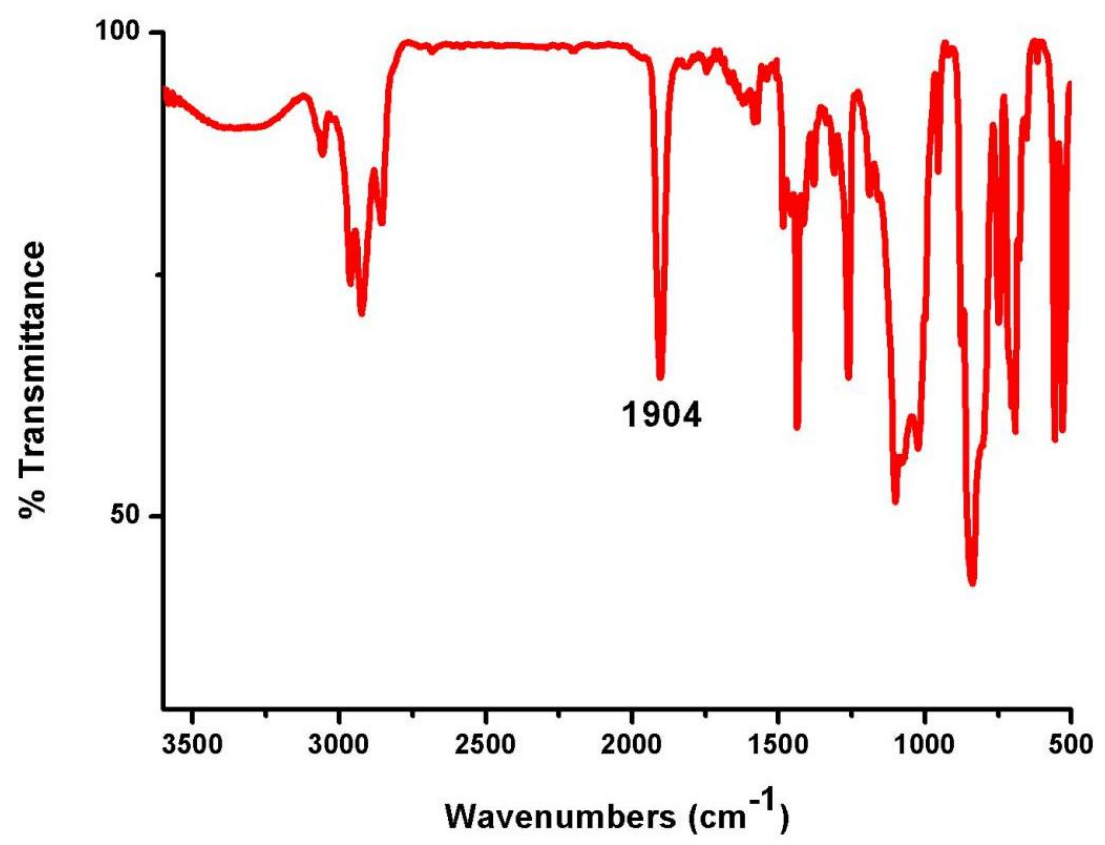

Figure S24. The IR (KBr) spectrum of 6

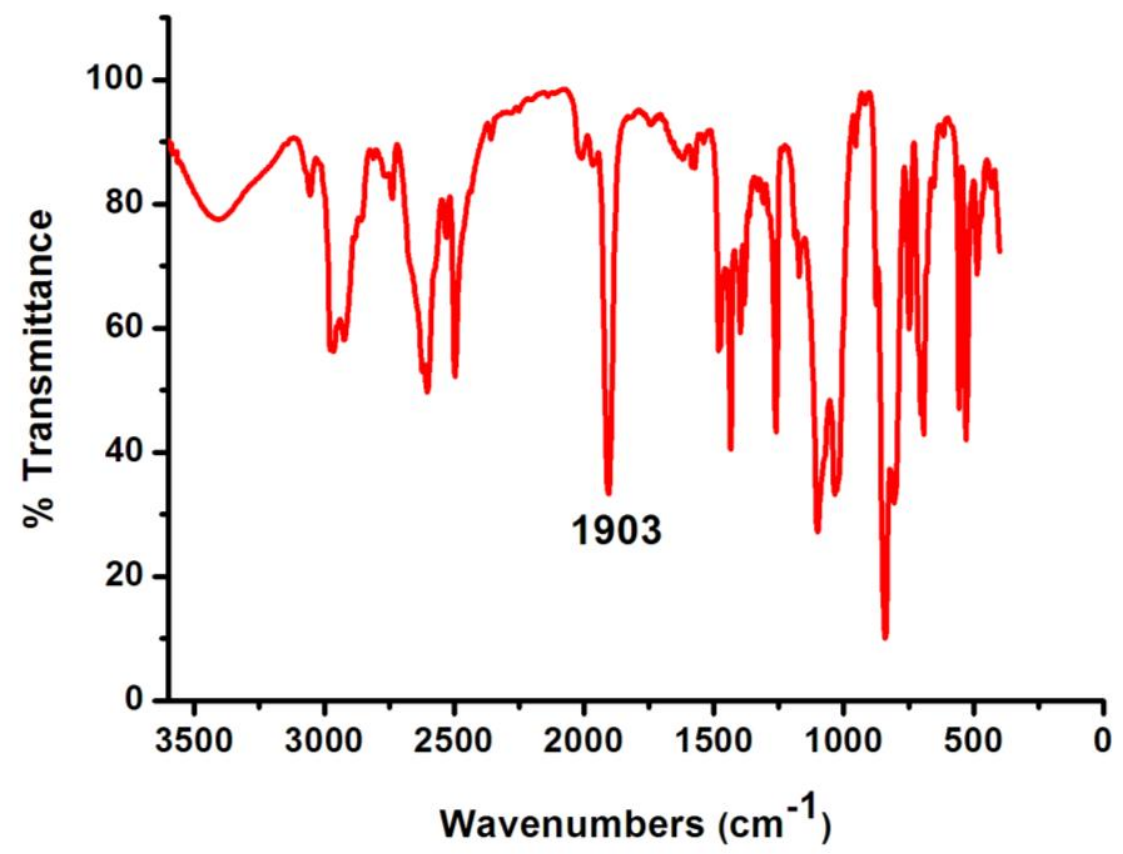


Figure S25. The cyclic voltammogram of $1(1 \mathrm{mM})$ in $0.1 \mathrm{M}^{n} \mathrm{Bu}_{4} \mathrm{NPF}_{6} / \mathrm{CH}_{2} \mathrm{Cl}_{2}$ at $25^{\circ} \mathrm{C}$ with a scan rate of $100 \mathrm{mV} \mathrm{s}^{-1}$

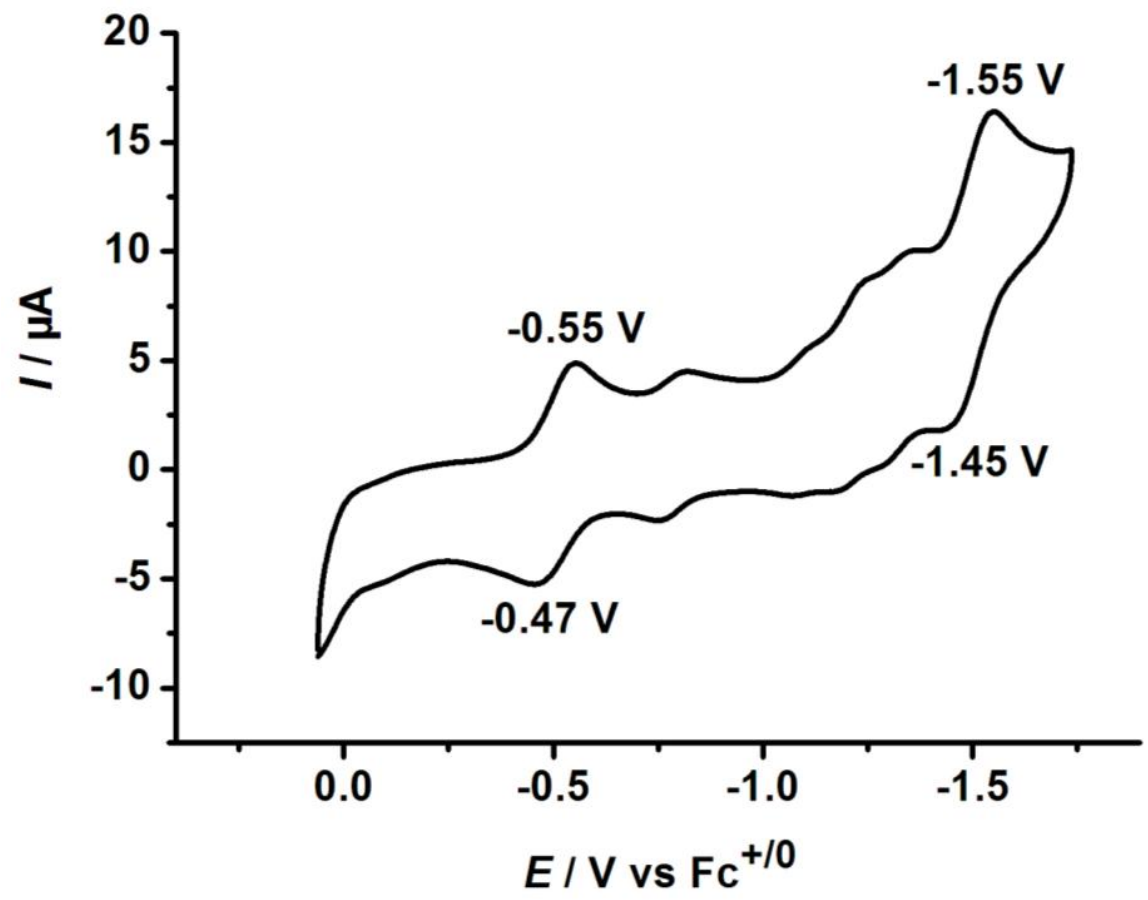

Figure S26. The cyclic voltammograms of $1(1 \mathrm{mM})$ in $0.1 \mathrm{M}^{n} \mathrm{Bu}_{4} \mathrm{NPF}_{6} / \mathrm{CH}_{2} \mathrm{Cl}_{2}$ at $25^{\circ} \mathrm{C}$ at various scan rates $\left(0.1 \mathrm{~V} \mathrm{~s}^{-1} \sim 1 \mathrm{~V} \mathrm{~s}^{-1}\right)$

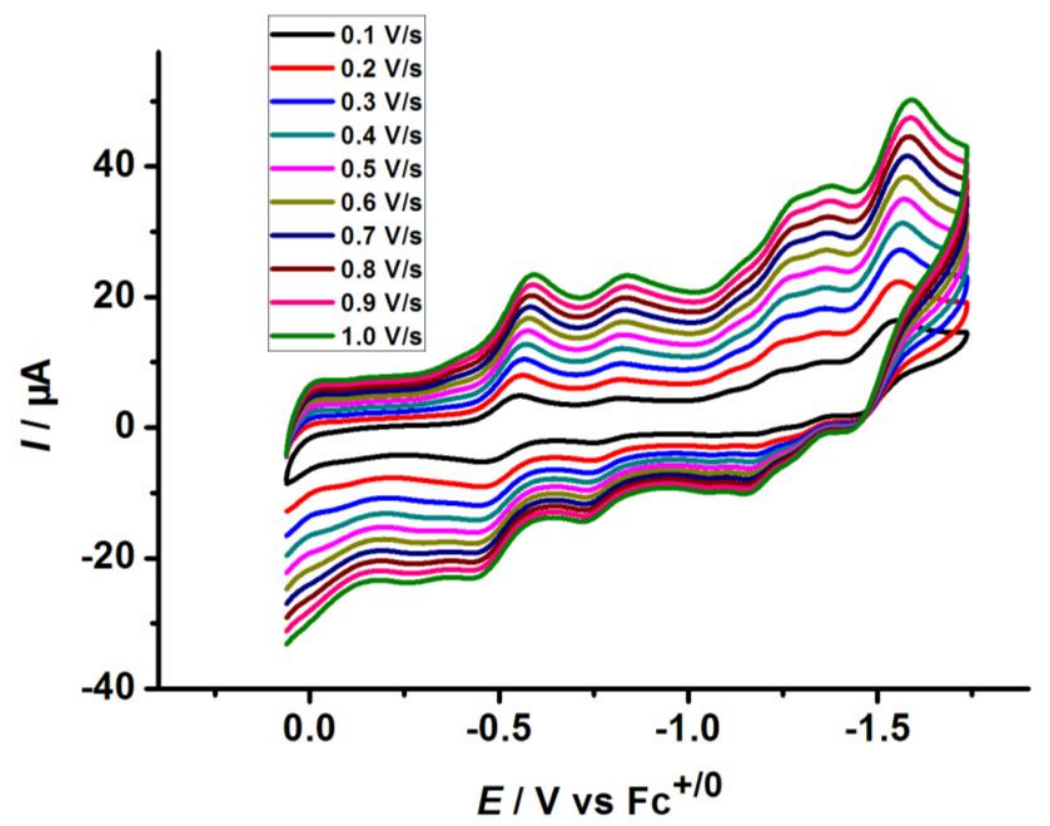


Figure S27. The cyclic voltammogram of $\mathbf{1}$ in the presence of 1 equivalent of $\left[\mathrm{HEt}_{3} \mathrm{~N}\right]\left[\mathrm{BF}_{4}\right]$ in $0.1 \mathrm{M}^{n} \mathrm{Bu}_{4} \mathrm{NPF}_{6} / \mathrm{CH}_{2} \mathrm{Cl}_{2}$ at $25^{\circ} \mathrm{C}$ with a scan rate of $100 \mathrm{mV} \mathrm{s}^{-1}$.

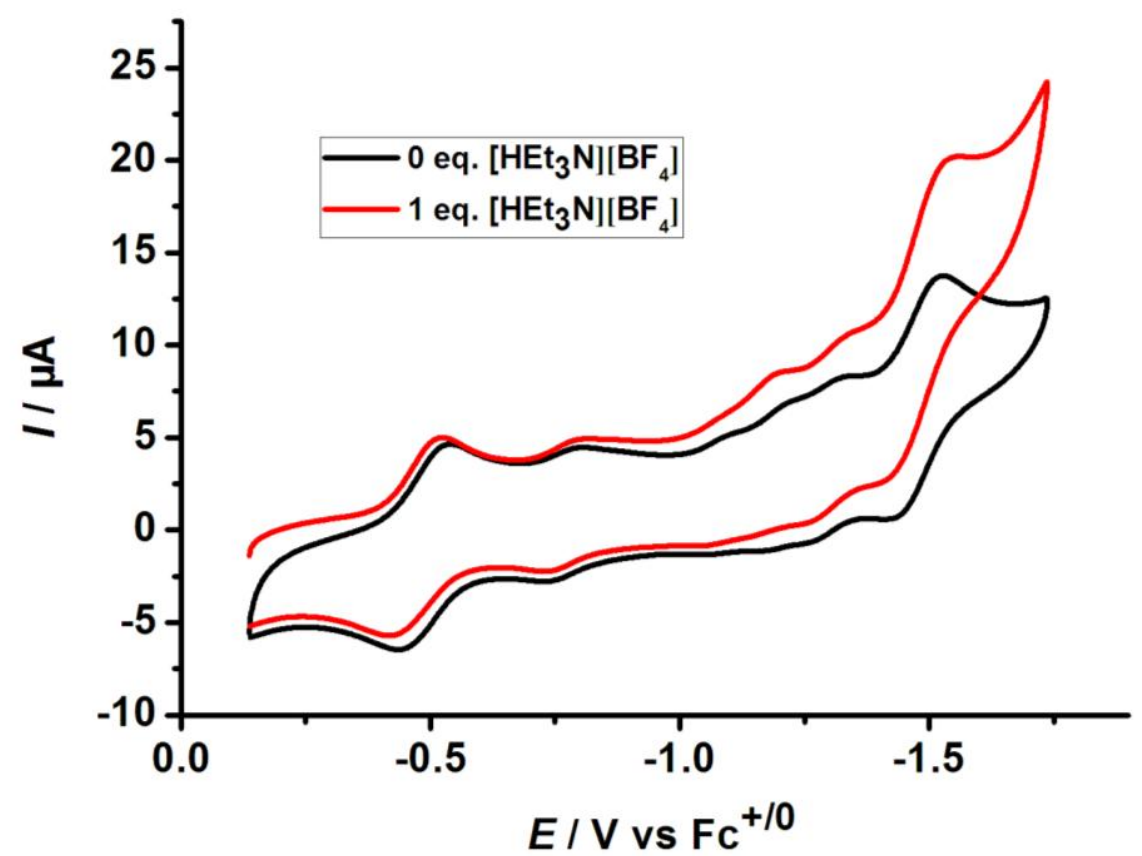

Figure S28. Cyclic voltammograms of $1\left(1 \mathrm{mM}\right.$ in $0.1 \mathrm{M}^{n} \mathrm{Bu}_{4} \mathrm{NPF}_{6}$ in $\mathrm{CH}_{2} \mathrm{Cl}_{2}$ under $\left.\mathrm{Ar}\right)$ with increments of $\left[\mathrm{HEt}_{3} \mathrm{~N}\right]\left[\mathrm{BF}_{4}\right](0,1,5,10,15,20 \mathrm{mM})$.

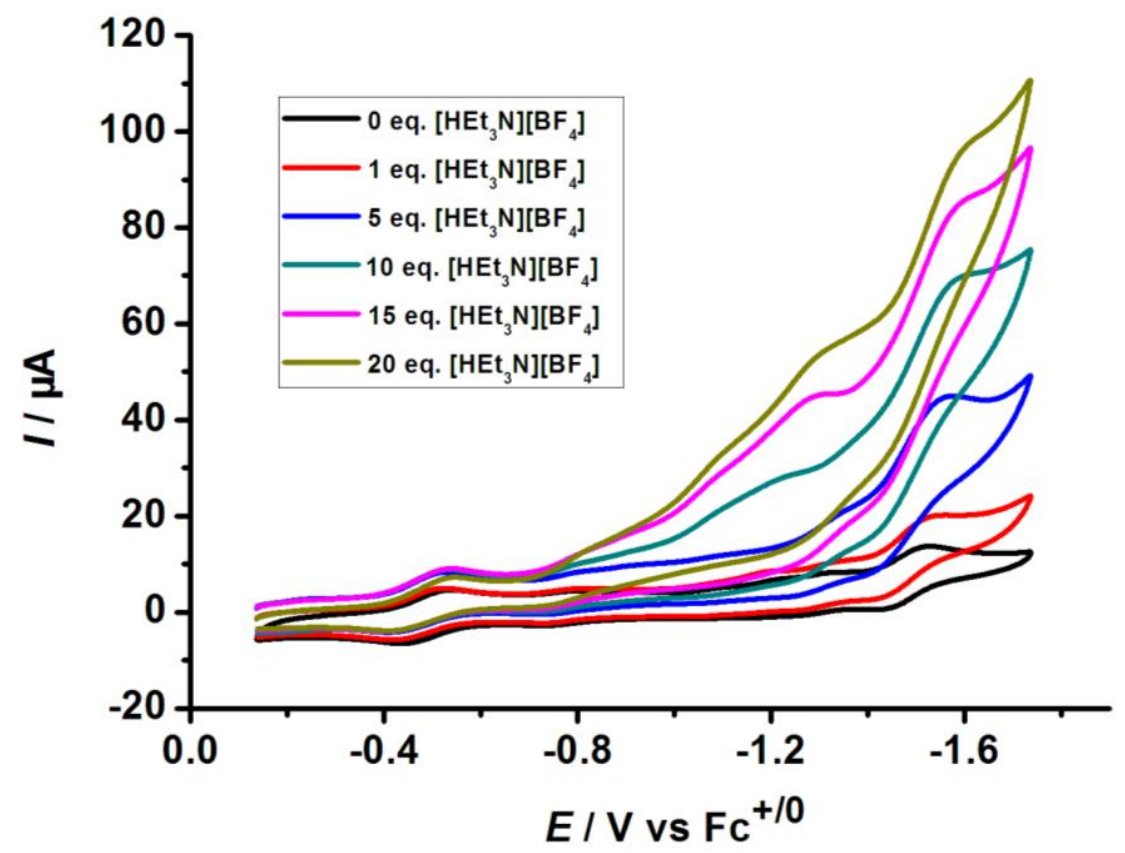


Figure S29. The cyclic voltammogram of $2(1 \mathrm{mM})$ in $0.1 \mathrm{M}^{n} \mathrm{Bu}_{4} \mathrm{NPF}_{6} / \mathrm{CH}_{2} \mathrm{Cl}_{2}$ at $25{ }^{\circ} \mathrm{C}$ with a scan rate of $100 \mathrm{mV} \mathrm{s}^{-1}$

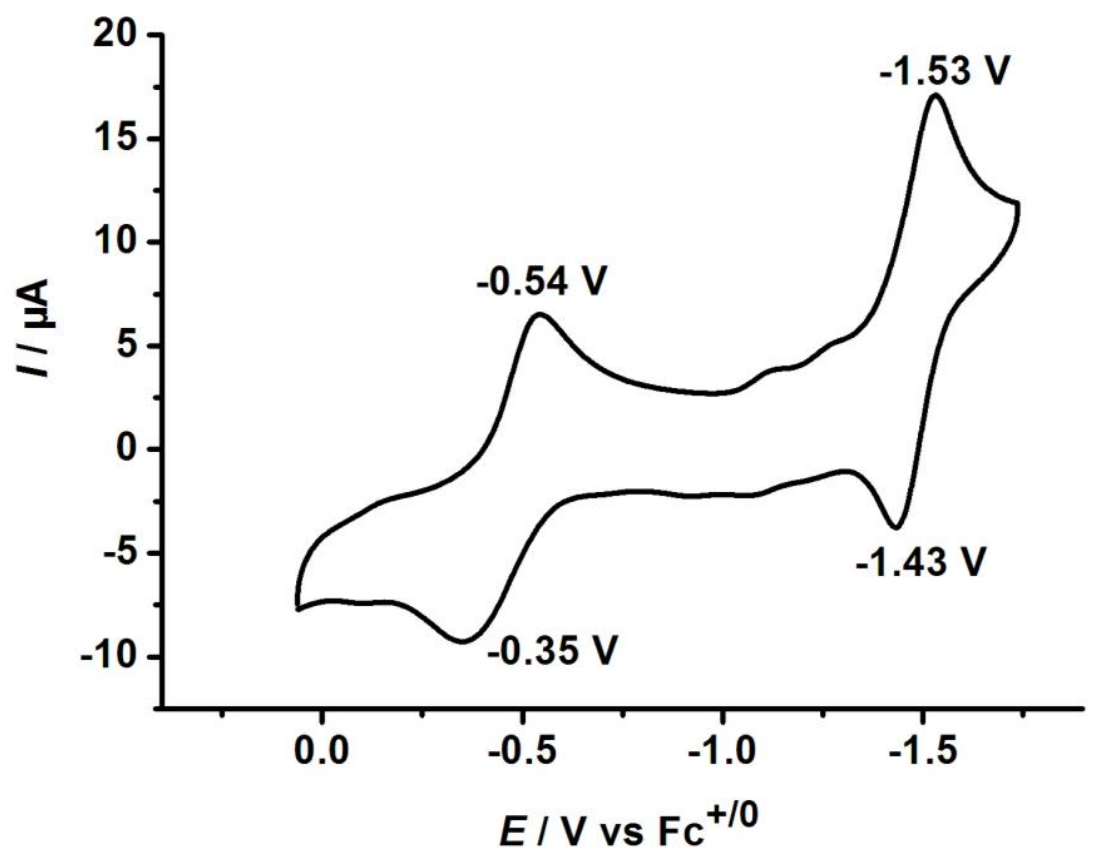

Figure S30. The cyclic voltammograms of $2(1 \mathrm{mM})$ in $0.1 \mathrm{M}^{n} \mathrm{Bu}_{4} \mathrm{NPF}_{6} / \mathrm{CH}_{2} \mathrm{Cl}_{2}$ at $25^{\circ} \mathrm{C}$ at various scan rates $\left(0.1 \mathrm{~V} \mathrm{~s}^{-1} \sim 1 \mathrm{~V} \mathrm{~s}^{-1}\right)$

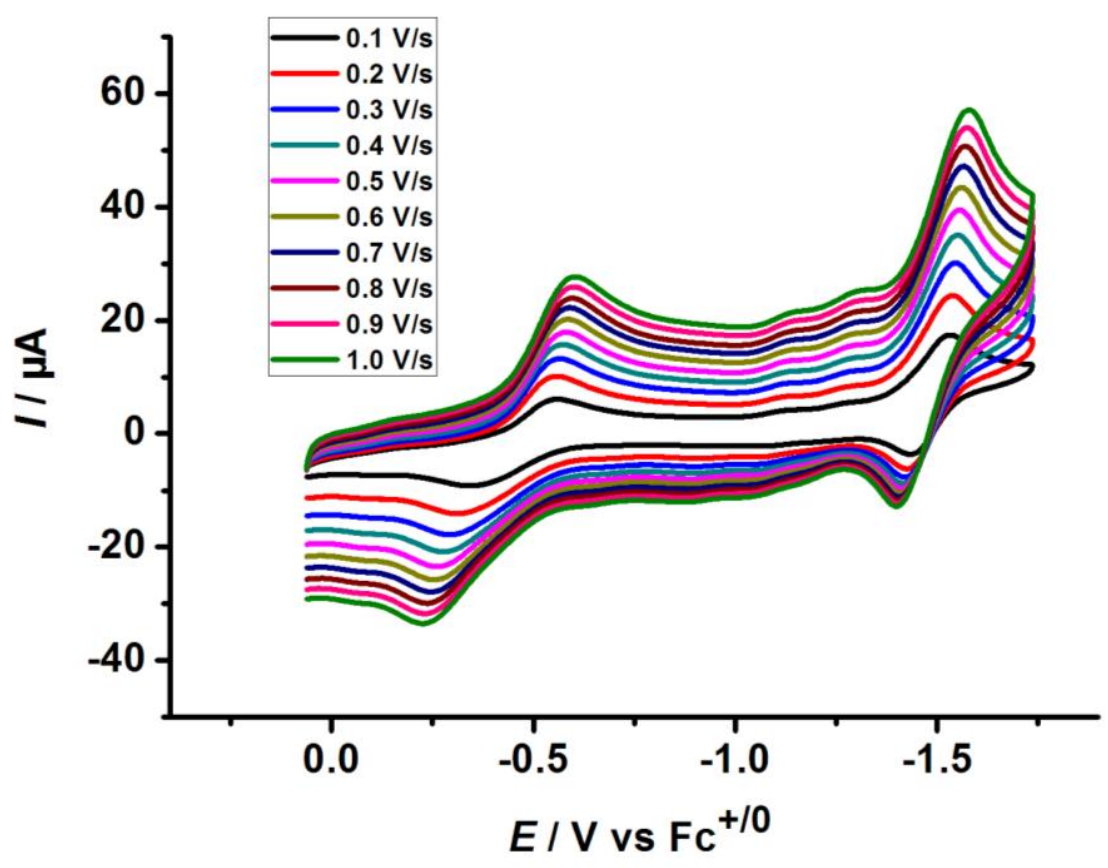


Figure S31. The cyclic voltammogram of 2 in the presence of 1 equivalent of $\left[\mathrm{HEt}_{3} \mathrm{~N}\right]\left[\mathrm{BF}_{4}\right]$ in $0.1 \mathrm{M}^{n} \mathrm{Bu}_{4} \mathrm{NPF}_{6} / \mathrm{CH}_{2} \mathrm{Cl}_{2}$ at $25^{\circ} \mathrm{C}$ with a scan rate of $100 \mathrm{mV} \mathrm{s}^{-1}$.

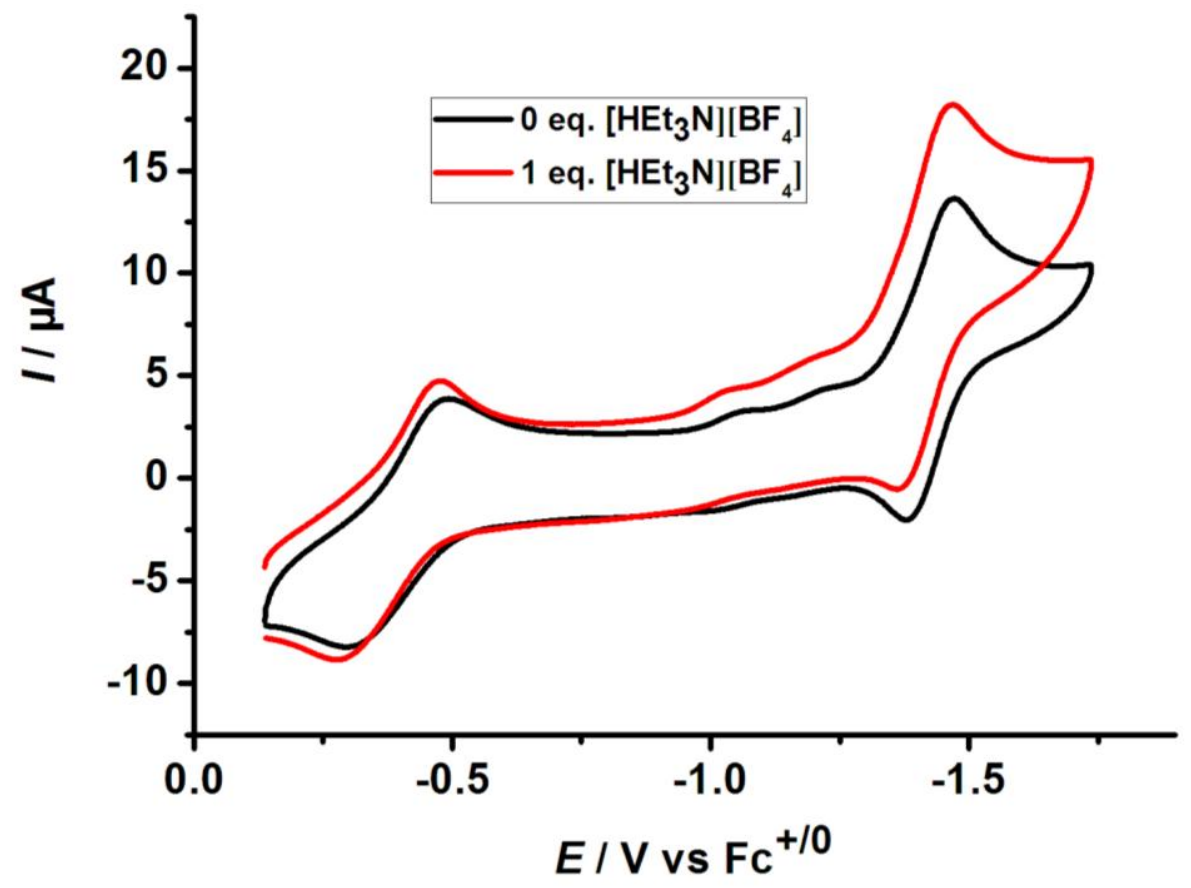

Figure S32. The cyclic voltammogram of $3(1 \mathrm{mM})$ in $0.1 \mathrm{M}^{n} \mathrm{Bu}_{4} \mathrm{NPF}_{6} / \mathrm{CH}_{2} \mathrm{Cl}_{2}$ at $25{ }^{\circ} \mathrm{C}$ with a scan rate of $100 \mathrm{mV} \mathrm{s}^{-1}$

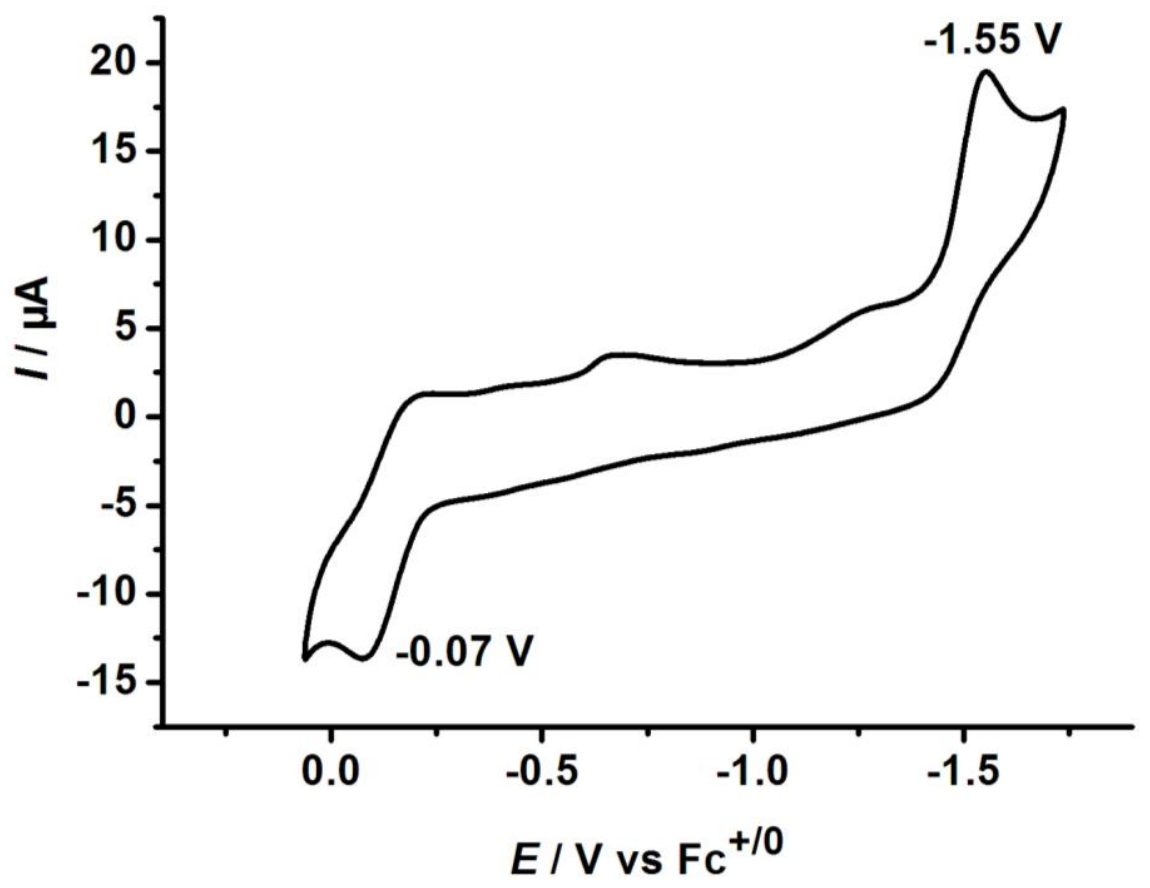


Figure S33. The cyclic voltammograms of $3(1 \mathrm{mM})$ in $0.1 \mathrm{M}^{n} \mathrm{Bu}_{4} \mathrm{NPF}_{6} / \mathrm{CH}_{2} \mathrm{Cl}_{2}$ at $25^{\circ} \mathrm{C}$ at various scan rates $\left(0.1 \mathrm{~V} \mathrm{~s}^{-1} \sim 1 \mathrm{~V} \mathrm{~s}^{-1}\right)$

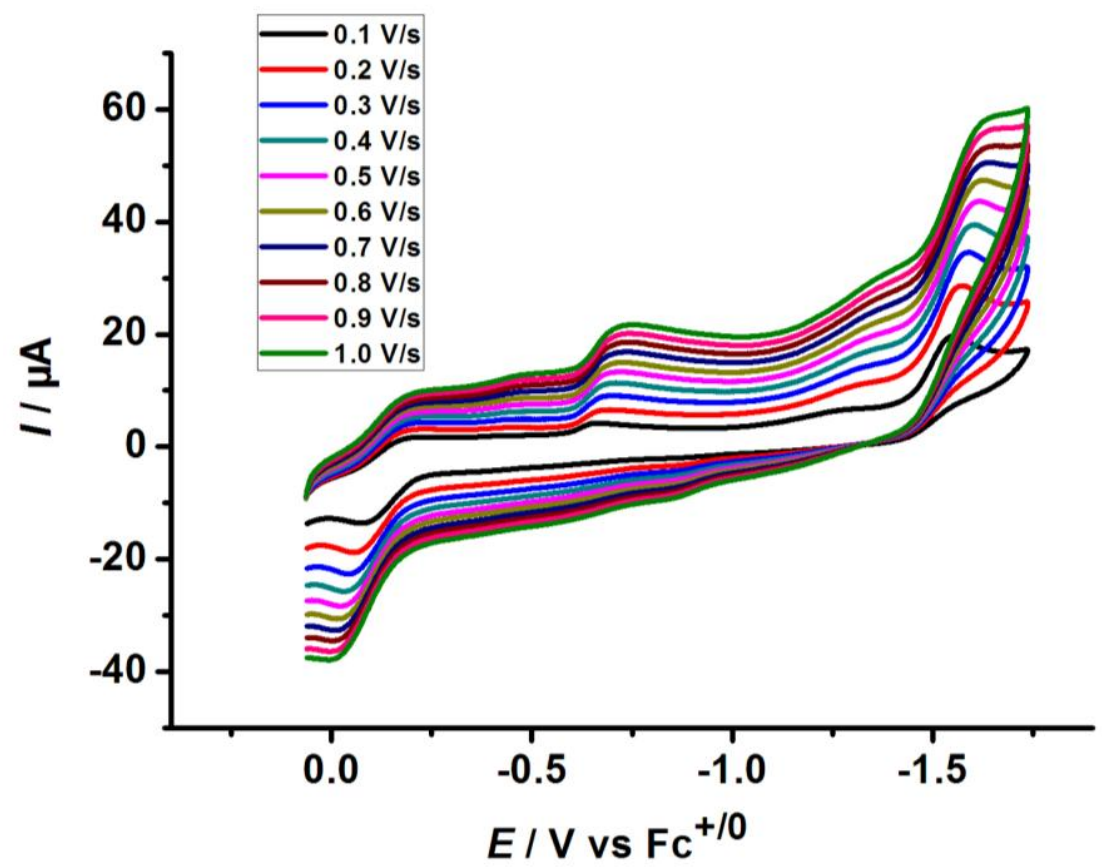

Figure S34. The cyclic voltammogram of $4(1 \mathrm{mM})$ in $0.1 \mathrm{M}^{n} \mathrm{Bu}_{4} \mathrm{NPF}_{6} / \mathrm{CH}_{2} \mathrm{Cl}_{2}$ at $25^{\circ} \mathrm{C}$ with a scan rate of $100 \mathrm{mV} \mathrm{s}^{-1}$

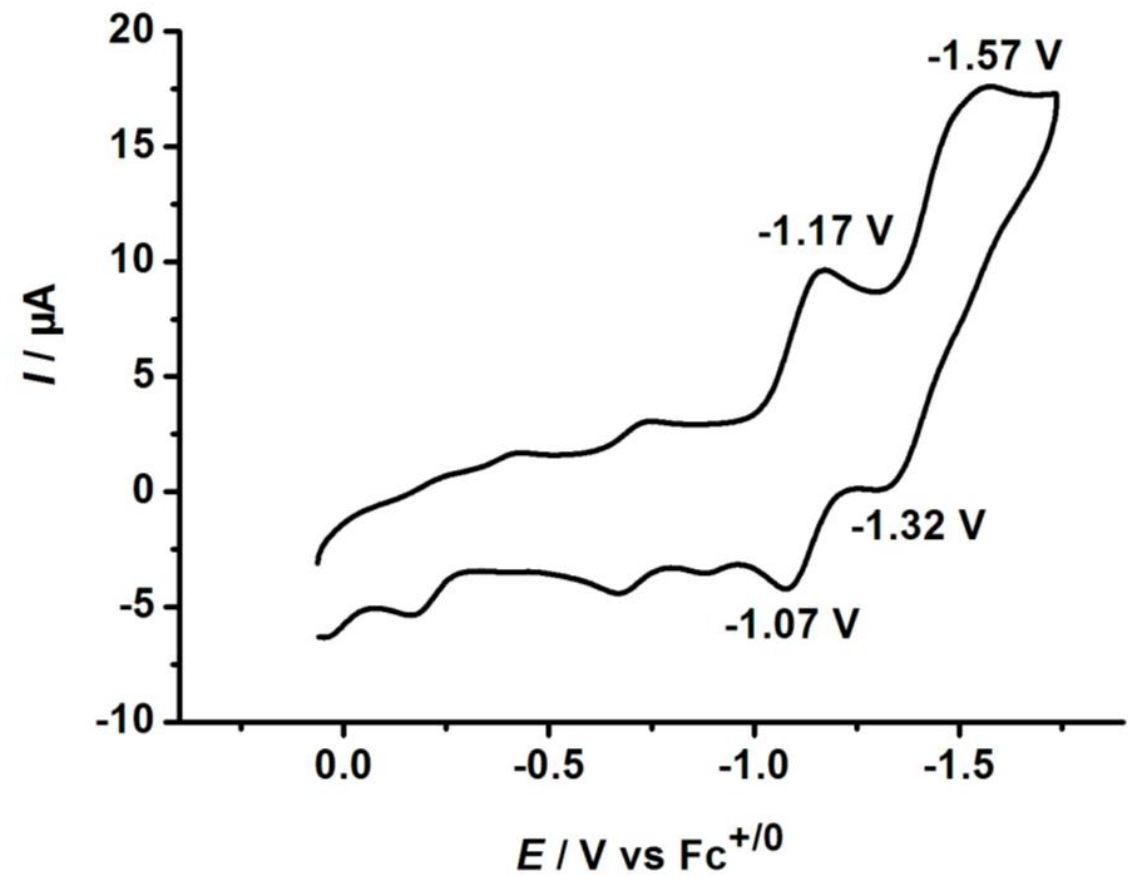


Figure S35. The cyclic voltammograms of $4(1 \mathrm{mM})$ in $0.1 \mathrm{M}^{n} \mathrm{Bu}_{4} \mathrm{NPF}_{6} / \mathrm{CH}_{2} \mathrm{Cl}_{2}$ at $25^{\circ} \mathrm{C}$ at various scan rates $\left(0.1 \mathrm{~V} \mathrm{~s}^{-1} \sim 1 \mathrm{~V} \mathrm{~s}^{-1}\right)$

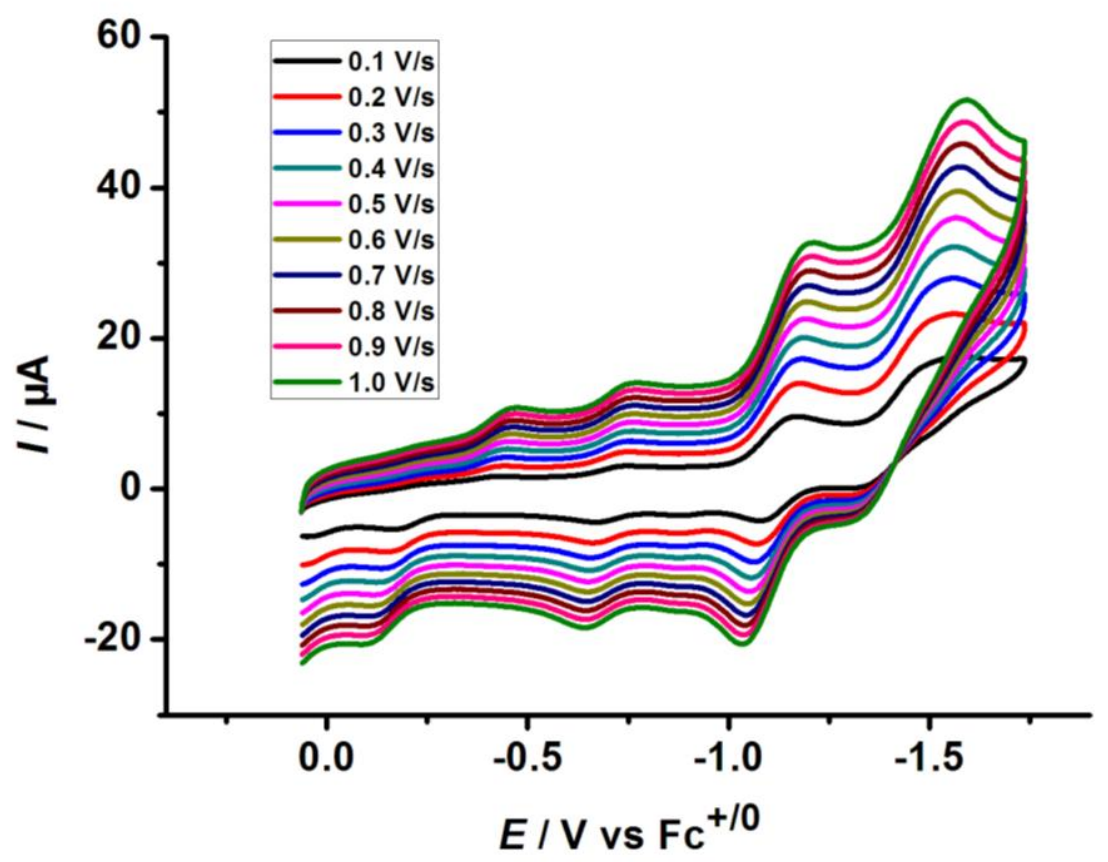

Figure S36. The cyclic voltammogram of $5(1 \mathrm{mM})$ in $0.1 \mathrm{M}^{n} \mathrm{Bu}_{4} \mathrm{NPF}_{6} / \mathrm{CH}_{2} \mathrm{Cl}_{2}$ at $25{ }^{\circ} \mathrm{C}$ with a scan rate of $100 \mathrm{mV} \mathrm{s}^{-1}$

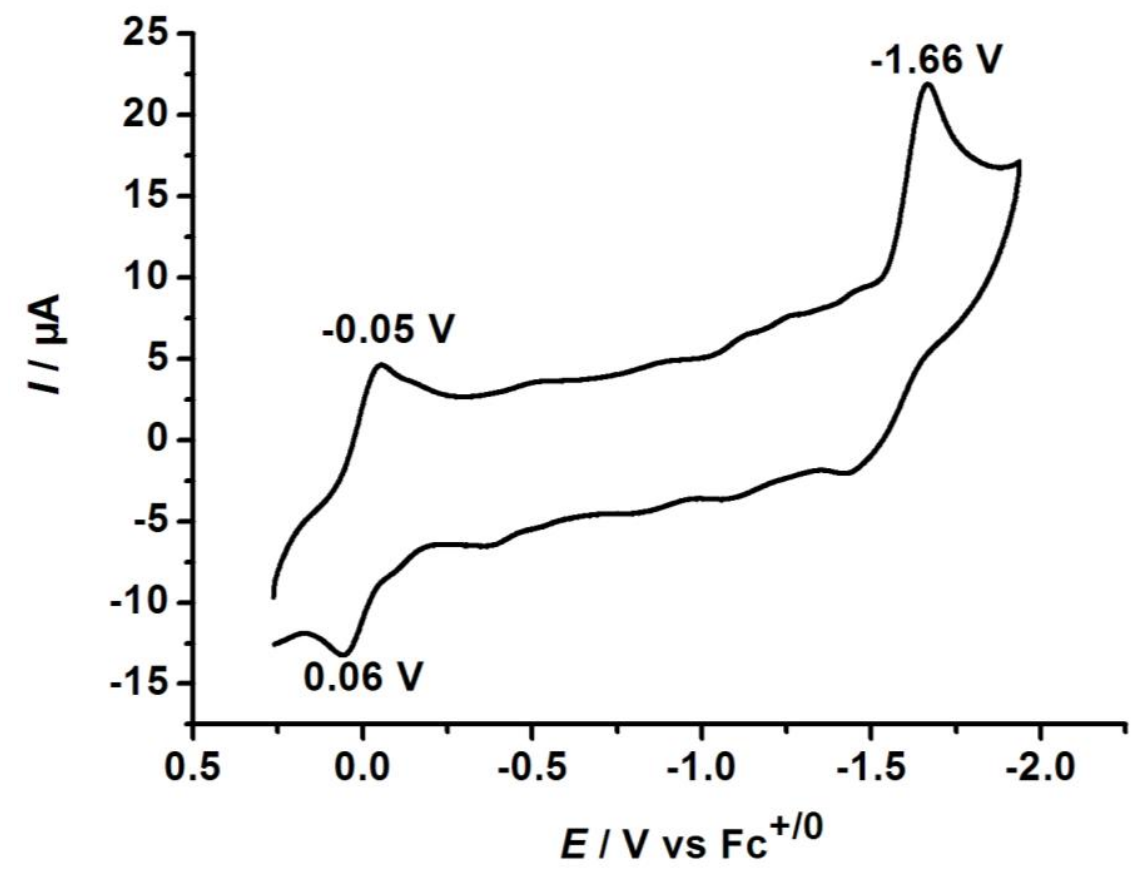


Figure S37. The cyclic voltammogram of $6(1 \mathrm{mM})$ in $0.1 \mathrm{M}^{n} \mathrm{Bu}_{4} \mathrm{NPF}_{6} / \mathrm{CH}_{2} \mathrm{Cl}_{2}$ at $25^{\circ} \mathrm{C}$ with a scan rate of $100 \mathrm{mV} \mathrm{s}^{-1}$

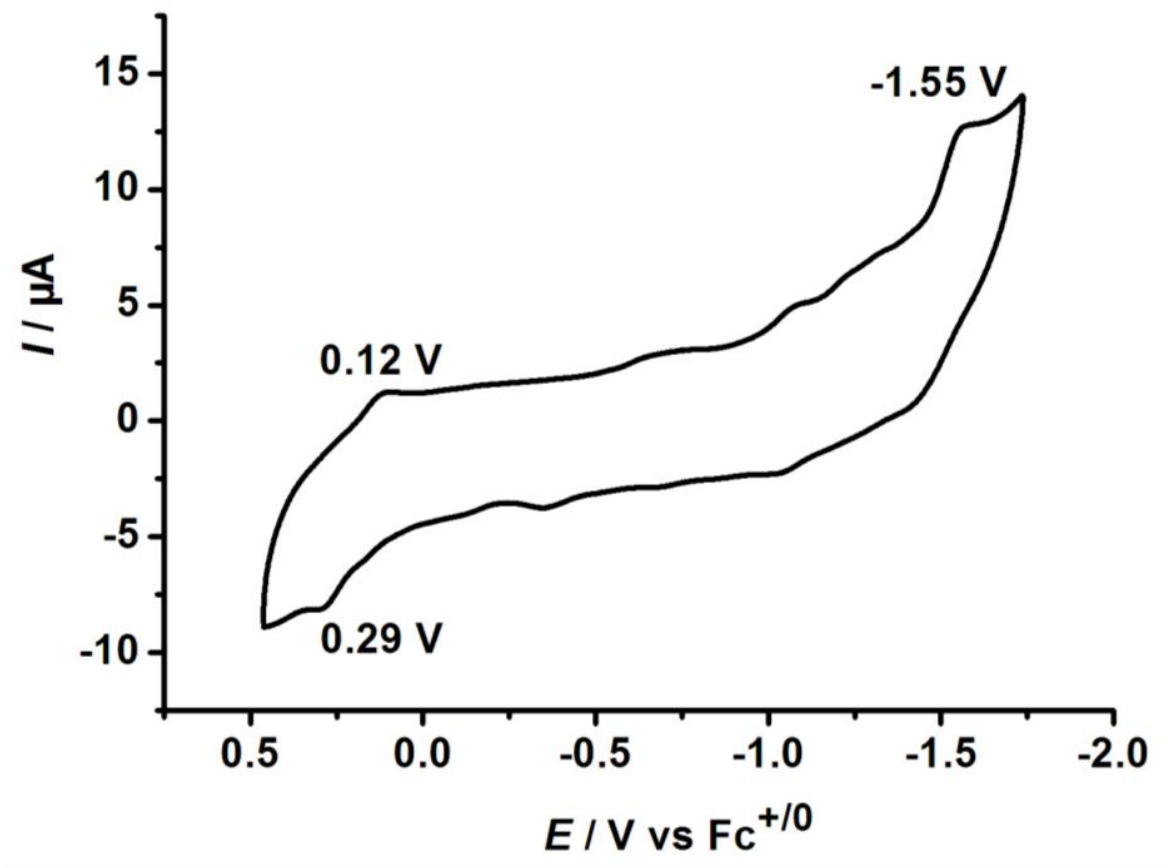

Figure S38. Corresponding plot of $\mathrm{i}_{\mathrm{c}} / \mathrm{i}_{\mathrm{p}}$ vs acid concentration

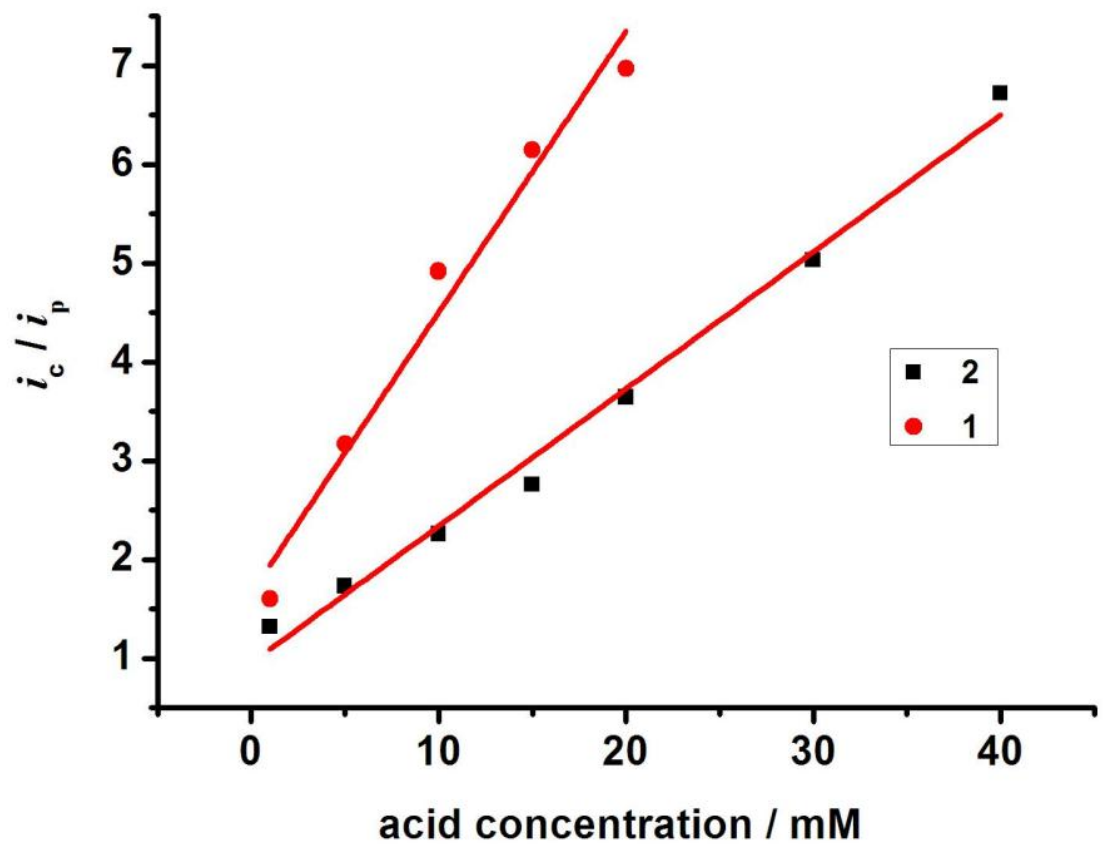

